\title{
Efficacy of a Single-Session "Empowered Relief" Zoom-Delivered Group Intervention for Chronic Pain: Randomized Controlled Trial Conducted During the COVID-19 Pandemic
}

Maisa S Ziadni ${ }^{1}$, MS, PhD; Lluvia Gonzalez-Castro ${ }^{1}$, BS; Steven Anderson ${ }^{1}, \mathrm{PhD}$; Parthasarathy Krishnamurthy ${ }^{2}$, $\mathrm{PhD}$; Beth D Darnall ${ }^{1}, \mathrm{PhD}$

\footnotetext{
${ }^{1}$ Department of Anesthesiology, Perioperative and Pain Medicine, Stanford University School of Medicine, Palo Alto, CA, United States

${ }^{2}$ C.T. Bauer College of Business, University of Houston, Houston, TX, United States
}

\section{Corresponding Author:}

Maisa S Ziadni, MS, PhD

Department of Anesthesiology, Perioperative and Pain Medicine

Stanford University School of Medicine

1070 Arastradero Road

Palo Alto, CA, 94304

United States

Phone: 16507365494

Email: mziadni@stanford.edu

\section{Abstract}

Background: Cognitive behavioral therapy-pain is an evidence-based treatment for chronic pain that can have significant patient burden, including health care cost, travel, multiple sessions, and lack of access in remote areas.

Objective: The study aims to pilot test the efficacy of a single-session videoconference-delivered empowered relief (ER) intervention compared to waitlist control (WLC) conditions among individuals with chronic pain. We hypothesized that ER would be superior to WLC in reducing pain catastrophizing, pain intensity, and other pain-related outcomes at 1-3 months posttreatment.

Methods: We conducted a randomized controlled trial involving a web-based sample of adults ( $\mathrm{N}=104)$ aged 18-80 years with self-reported chronic pain. Participants were randomized (1:1) to 1 of 2 unblinded study groups: ER (50/104, 48.1\%) and WLC (54/104, 51.9\%). Participants allocated to ER completed a Zoom-delivered class, and all participants completed follow-up surveys at 2 weeks and 1,2, and 3 months posttreatment. All the study procedures were performed remotely and electronically. The primary outcome was pain catastrophizing 1-month posttreatment, with pain intensity, pain bothersomeness, and sleep disruption as secondary outcomes. We also report a more rigorous test of the durability of treatment effects at 3 months posttreatment. Data were collected from September 2020 to February 2021 and analyzed using intention-to-treat analysis. The analytic data set included participants (18/101, 17.8\% clinic patients; 83/101, 82.1\% community) who completed at least one study survey: ER $(50 / 101,49.5 \%)$ and WLC $(51 / 104,49 \%)$.

Results: Participants (N=101) were 69.3\% (70/101) female, with a mean age of 49.76 years (SD 13.90; range 24-78); 32.7\% (33/101) had an undergraduate degree and self-reported chronic pain for 3 months. Participants reported high engagement $(47 / 50$, 94\%), high satisfaction with ER (mean 8.26, SD 1.57; range 0-10), and high satisfaction with the Zoom platform (46/50, 92\%). For the between-groups factor, ER was superior to WLC for all primary and secondary outcomes at 3 months posttreatment (highest $P<.001$ ), and between-groups Cohen $d$ effect sizes ranged from 0.45 to 0.79 , indicating that the superiority was of moderate to substantial clinical importance. At 3 months, clinically meaningful pain catastrophizing scale (PCS) reductions were found for ER but not for WLC (ER: PCS -8.72, 42.25\% reduction; WLC: PCS -2.25, 11.13\% reduction). ER resulted in significant improvements in pain intensity, sleep disturbance, and clinical improvements in pain bothersomeness.

Conclusions: Zoom-delivered ER had high participant satisfaction and very high engagement. Among adults with chronic pain, this single-session, Zoom-delivered, skills-based pain class resulted in clinically significant improvement across a range of pain-related outcomes that was sustained at 3 months. Web-based delivery of ER could allow greater accessibility of home-based pain treatment and could address the inconveniences and barriers faced by patients when attempting to receive in-person care.

Trial Registration: ClinicalTrials.gov NCT04546685; https://clinicaltrials.gov/ct2/show/NCT04546685 
(J Med Internet Res 2021;23(9):e29672) doi: 10.2196/29672

\section{KEYWORDS}

single-session; empowered relief; Zoom-delivered; pain catastrophizing; pain intensity; randomized-controlled trial; chronic pain

\section{Introduction}

\section{Background}

Chronic pain is a significant public health concern. It is one of the most common reasons for seeking medical care [1], with considerable societal and financial burden $[2,3]$ in addition to human suffering. Chronic pain treatment is challenging due to limitations of existing pharmacological approaches, particularly intolerability of many medicines [4], health risks [5-9], and lack of sustainable availability. In contrast, behavioral medicine approaches are low risk and have been shown to have low-to-moderate efficacy for a range of chronic pain conditions [5,7,10-12], with cognitive behavioral therapy (CBT)-pain as the established evidence-based treatment for chronic pain [8,9]. However, several barriers prevent broad access to CBT-pain, including out-of-pocket costs, burdensome travel, lack of availability in remote or rural areas, and a lack of adequately trained clinicians $[6,13]$. The length of treatment (ie, eight sessions or more for CBT-pain) is associated with increased dropout [14], which may perpetuate the overuse of medical services and health disparities. The COVID-19 pandemic has further challenged the feasibility of multisession in-person treatment options [15], and this creates the need for accessible solutions that are low-cost, low-burden, and remove the need for face-to-face meetings [16].

Brief web-based and remotely delivered psychological interventions have been demonstrated to accomplish this goal and have been found to be as effective as face-to-face therapy, while decreasing health care use and barriers to treatment [17]. Effective web-based treatments now exist for various psychological disorders, including depression, panic disorder, and social phobia [18]. Web-based CBT has been effective for a number of chronic health conditions, including irritable bowel syndrome, tinnitus, and headache [19,20]. In a systematic review of web-based interventions for chronic back pain, Garg et al [21] reported that web-based interventions, including CBT, effectively reduced pain catastrophizing scale (PCS) scores. Effect sizes for web-based interventions for chronic pain have been estimated to range from small to moderate (Cohen $d$ range 0.04-1.23) [18]. In addition to CBT, existing web-based interventions for patients with pain include compassionate mind training [22], social media-based web-based community intervention [23], pain self-management [24], and hypnosis [25]. Despite the promise of web-based interventions for pain, multisession treatments typically range from 20 days to 12 weeks in duration, which can be costly, burdensome, and limit access to adequate care [26].

Single-session interventions (SSIs), which have been defined as the intentional delivery of a specific, structured program involving a single visit with a clinic, provider, or program [27], have the potential to reduce the burden of traditional multisession treatment approaches and expand access to behavioral medicine. SSIs have been found to be effective for a variety of psychological and health conditions, including severe mental illness [28], anxiety and youth conduct disorder [27], acute insomnia [29], heavy alcohol consumption in college students [30], trauma and adversity [31,32], postsurgical pain [33], and chronic pain [11,12,34-36]. SSIs that have been further optimized via web-based delivery have demonstrated feasibility and efficacy similar to face-to-face interventions $[11,12,37]$ and include interventions for multiple sclerosis pain [38], disordered gambling [39], and adolescent mental health [40-42]. In addition to reducing treatment burdens, web-based SSIs have the vital advantage of ease of scalability, as they can be completed by patients in any location [37]. Effective web and digital-based SSIs would eliminate or reduce many of the existing barriers to treatment, such as cost, lack of trained therapists, and insurance limits on the length of treatment. However, despite the promise of SSIs, rigorously designed and randomized controlled studies are needed to establish their efficacy [43].

\section{Objectives}

Building on the limited literature on web-based SSIs for chronic pain, we aim to evaluate a Zoom-delivered version of a previously developed and efficacious, skills-based, behavioral medicine SSI empowered relief (ER) [11,36]. Previously, our group conducted a three-arm randomized controlled trial conducted in 263 adults with chronic low back pain comparing in-person ER to a health education class or 8-week CBT [12]. However, web-based delivery of ER has not been tested, particularly in mixed-etiology chronic pain, and the COVID-19 context underscores the importance of providing effective home-based chronic pain care. Extending this work to the digital platform, in this study, we conducted a parallel-group, randomized (1:1) comparative efficacy trial to assess the impact of a single-session videoconference-delivered ER group pain relief skills class versus waitlist control (WLC). We hypothesize that at 3 months posttreatment, (1) ER would be superior to WLC for reductions in pain catastrophizing, an index of pain coping; (2) ER would be superior to WLC for reductions in pain intensity, bothersomeness, and sleep disturbance; and (3) ER would be superior to WLC in reducing anxiety, depression, and physical function.

\section{Methods}

\section{Overview and Setting}

This study was a parallel-group, randomized, clinical trial comparing ER, a 2-hour single-session videoconference-delivered class, to a WLC. Enrolled participants $(\mathrm{N}=104)$ aged $18-80$ years with mixed chronic pain etiology were randomized $(1: 1)$ to one of the two study arms. Participants completed outcome assessments at pretreatment, week 2, and 1, 2, and 3 months posttreatment. The primary outcome was pain catastrophizing levels at the 1-month follow-up [35], with pain intensity, pain, bothersomeness, and sleep disturbance as secondary outcomes. The tertiary outcomes 
included PROMIS (Patient-Reported Outcomes Measurement Information System) measures of anxiety, depression, and physical function at the 1-month follow-up time-point. We also report a more rigorous test of the durability of treatment effects at 3 months posttreatment as highlighted in the Results section. A crossover intervention class was offered to participants who were initially randomized to the WLC and was conducted in February 2021. Of 48 participants who were offered the treatment, 20 (42\%) attended the crossover class at no cost, and no data were collected. This study was conducted between August 2020 and February 2021.

\section{Study Sample, Recruitment, and Participant Compensation}

Participants were recruited remotely through targeted emails to lists of patients who agreed to be contacted for research purposes through two databases: (1) the Stanford's Collaborative Health Outcomes Information Registry comprising patients who have received care at the Stanford Pain Management Center (a tertiary referral pain clinic in Redwood City, California) and (2) the Stanford Systems Neuroscience and Pain Lab database of individuals with chronic pain. Interested individuals were directed to a web-based screening form, and those who met the initial eligibility criteria were contacted by research staff by phone to confirm their eligibility. Approximately $18.3 \%$ $(19 / 104)$ of the study sample were clinic patients, and $81.7 \%$ $(85 / 104)$ were from the larger community. Eligible and interested participants were invited to participate in a pain-skills treatment class compared with a WLC and completed an electronic informed consent form (see Multimedia Appendix 1 for the study consent form). After informed consent was obtained, study participants were randomized one-to-one using a REDCap (Research Electronic Data Capture; Vanderbilt University) [44] cloud random number generator and allocated to the study group. All study procedures were completed remotely, and no in-person visits were required. Figure 1 displays the participants' study activities.

Figure 1. Participant activities. ER: empowered relief; WLC: waitlist control.

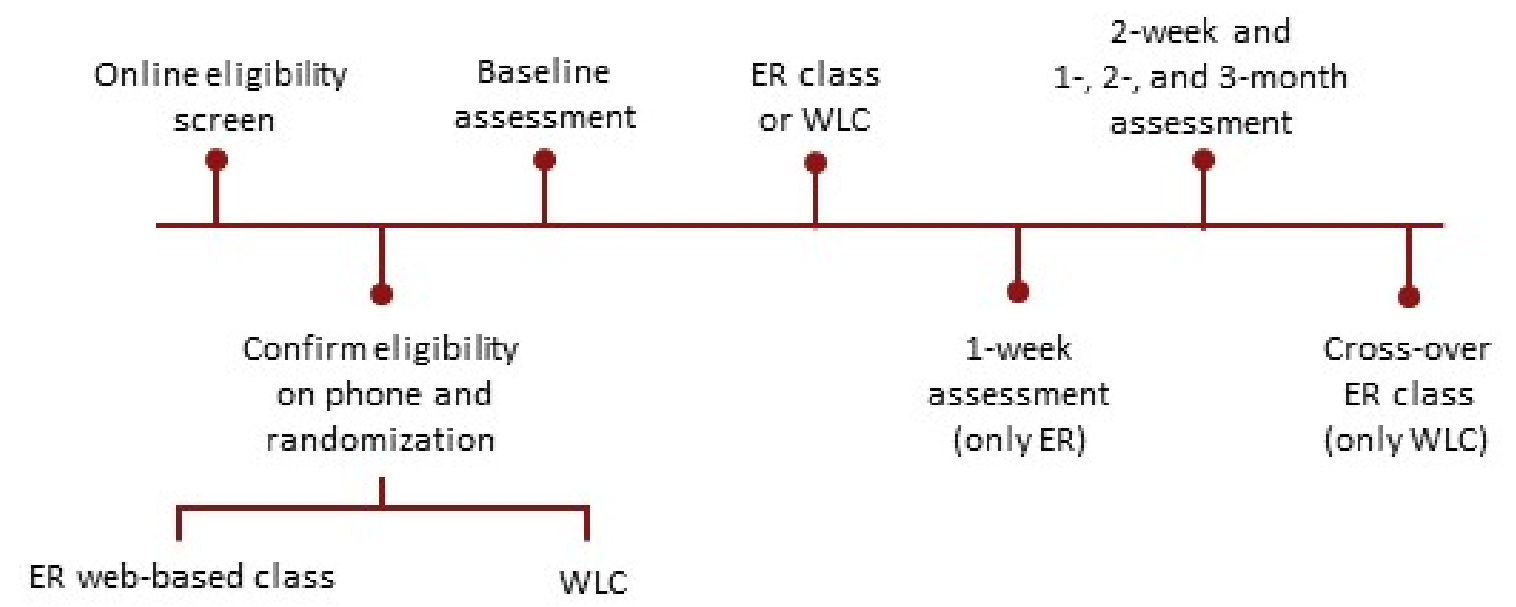

All the study procedures were performed remotely and electronically. Enrolled participants completed a baseline survey, which was conducted 4 days before the scheduled class, followed by posttreatment surveys at 2 weeks, and 1, 2, and 3 months. The intervention was conducted on Zoom [45], and details of the Zoom session were emailed to participants before the scheduled class time. Overall, in the ER arm, two cohorts were recruited, each consisting of 24-26 participants per class. The class sizes were consistent with studies on web-based interventions [46-51]. Participants received US \$10 in the form of an Amazon electronic gift code for each survey they completed and could receive up to US $\$ 50$ following completion of all study procedures. The research procedures were approved by the Stanford institutional review board.

\section{Inclusion and Exclusion Criteria}

All study recruitment efforts directed interested individuals with chronic pain to the web-based screening form to complete an automated eligibility form that screened for initial inclusion and exclusion criteria. Participants who met initial eligibility were then contacted by the research staff to confirm study eligibility according to the inclusion and exclusion criteria provided in Textbox 1. Internet and computer literacy were the implicit de facto eligibility criteria. If interested participants indicated that they had never used the Zoom platform, the research coordinator contacted them by phone to schedule an individual meeting; their skill to participate in the web-based class would increase because they were more familiar with the Zoom platform and navigating the tool in a supervised setting. Of the 9 participants who indicated that they had never used Zoom, none requested to schedule an individual meeting. 
Textbox 1. Inclusion and exclusion criteria.

Inclusion criteria
- $\quad$ Pain $>3$ months more than half the time
- $\quad$ English fluency
- $\quad$ Femality to attend a one-time web-based class (if assigned) and complete web-based surveys
Exclusion criteria
- $\quad$ Ongoing legal action or disability claim
- $\quad$ Previous participation in the empowered relief class
- Cognitive impairment, non-English-speaking, or psychological factors that would preclude comprehension of material or full participation in
the study
the study

\section{Randomization and Blinding}

Participants were randomized 1:1 (no blocking applied) and allocated to one of two study arms: (1) ER or (2) WLC. REDCap was used to apply an automatic and blinded randomization program and ensure equal allocation to both groups. Participants were not blinded to the study arm, given the nature of WLC. Statisticians performed blinded analysis of data sets that were randomly labeled as group 1 and group 2, with statistician unblinding occurring only after posttreatment month 3 data were analyzed. The study coordinator (LGC) was unblinded to the individual study arm assignments and was not involved in any data analyses. The study protocol was reviewed and approved [52].

\section{Study Groups \\ Single-Session Videoconference-Delivered Skills-Based Pain Class (ER)}

\section{Overview}

ER was developed in 2013, with pilot data [36] showing a reduction in pain catastrophizing 1-month posttreatment, despite comorbid emotional disorders. Notably, a National Institute of Health-funded, three-arm, randomized controlled trial [11,12] conducted in 263 individuals with chronic low back pain demonstrated that 3-month posttreatment, the ER class was equally effective to an 8-week CBT-pain intervention and was superior to a health education control class in reducing pain catastrophizing and pain intensity. ER compresses key elements and skills from CBT-pain and mindfulness into a single-session 2 -hour class $[11,12,34,36]$. The class is didactic and delivered with a standardized instructor slide deck and manual. Participants learn self-management skills to decrease physiological hyperarousal, which includes diaphragmatic breathing, cognitive reframing, and self-soothing strategies. During the class, participants self-tailor the skills content by completing a personalized plan for ER. They also received an MP3 audio file with a 20-minute version of the relaxed breathing exercise. The participants can download the audio file from a secure web-based storage platform onto their various devices (mobile or tablet) for convenience. The participants were encouraged to practice the learned skills daily.

\section{Class Platform}

The Zoom platform was used to deliver the ER classes, and instructors shared their PowerPoint presentation slides throughout the class. Class participants were encouraged to ask questions at any time through the Zoom chat box and unmute themselves to participate. Zoom is password-protected and hosted within the firewalled Stanford University School of Medicine and Stanford HealthCare systems.

\section{Instructors}

The class instructors were doctoral-level clinical psychologists certified by Stanford University [53] to deliver the intervention.

\section{Training and Monitoring of Instructors}

The instructors were familiar with the study protocol, were certified in ER delivery, and had extensive experience delivering the class. ER was developed to ensure fidelity (eg, standardized slide deck and instructor manual; instructor certification process), and cohort effects were minimal due to the single session and didactic nature of the intervention, with minimal participant interaction.

\section{Waitlist Control}

The study used a WLC group because the ER class web-based delivery is a new approach that has not been previously tested $[54,55]$. During the screening phone call, participants randomized to the WLC were advised to continue any ongoing clinical care. Although the WLC group did not receive any study intervention, participants were informed that they would be offered the ER treatment class after study completion at 3 months.

\section{Data Collection and Variable Measurement}

All surveys were collected through REDCap [44], a web-based electronic data capture platform, which is a secure (password protected), HIPAA (Health Insurance Portability and Accountability Act)-compliant platform, and hosted by the Stanford University School of Medicine.

Data collection consisted of electronically collected participant-reported measures across the four phases of the study: screening, pretreatment, treatment, and posttreatment. For both groups, posttreatment survey collection was conducted at 2 weeks and 1-3 months posttreatment. The ER group 
completed an additional brief survey 1-week posttreatment to assess participant satisfaction with the intervention and Zoom platform. Table 1 shows the data collection time-points for both the groups. Baseline surveys included demographic information, pain intensity, pain bothersomeness, pain catastrophizing, and PROMIS measures. Posttreatment surveys included the same measures, excluding the demographics.

Table 1. Timeline of variable assessment.

\begin{tabular}{|c|c|c|c|c|}
\hline \multirow[t]{2}{*}{ Variables } & \multirow{2}{*}{$\begin{array}{l}\text { Pretreatment } \\
\text { Baseline }\end{array}$} & \multicolumn{3}{|c|}{ Posttreatment follow-up } \\
\hline & & 1 week & 2 weeks & 1,2 , and 3 months \\
\hline Demographics and pain condition & $\mathfrak{S}^{\mathrm{a}}$ & & & \\
\hline $\mathrm{PCS}^{\mathrm{b}}$ & $\checkmark$ & & $\checkmark$ & $\checkmark$ \\
\hline Pain bothersomeness & $\checkmark$ & & $\checkmark$ & $\checkmark$ \\
\hline \multicolumn{5}{|l|}{ PROMIS $^{\mathrm{c}}$} \\
\hline Depression & $\checkmark$ & & $\checkmark$ & $\checkmark$ \\
\hline Anxiety & $\checkmark$ & & $\checkmark$ & $\checkmark$ \\
\hline Pain intensity & $\checkmark$ & & $\checkmark$ & $\checkmark$ \\
\hline Physical function & $\checkmark$ & & $\checkmark$ & $\checkmark$ \\
\hline Pain interference & $\checkmark$ & & $\checkmark$ & $\checkmark$ \\
\hline Fatigue & $\checkmark$ & & $\checkmark$ & $\checkmark$ \\
\hline Social isolation & $\checkmark$ & & $\checkmark$ & $\checkmark$ \\
\hline Anger & $\checkmark$ & & $\checkmark$ & $\checkmark$ \\
\hline Sleep disturbance & $\checkmark$ & & $\checkmark$ & $\checkmark$ \\
\hline Satisfaction with treatment & & $\checkmark$ & & \\
\hline
\end{tabular}

${ }^{\mathrm{a}}$ Variable assessed.

${ }^{\mathrm{b}} \mathrm{PCS}$ : pain catastrophizing scale.

${ }^{\mathrm{c}}$ PROMIS: Patient-Reported Outcomes Measurement Information System.

\section{Study Variables and Measures}

\section{Pain Catastrophizing}

The 13-item PCS [56] measures negative thoughts and emotional responses to pain. PCS is a $1 \mathrm{D}$ measurement with three subscales: helplessness, magnification, and rumination. It is scored by summing all items and generates a total score, with higher scores indicating greater catastrophizing. The PCS is a reliable, validated, and psychometrically trusted instrument [57].

\section{Pain Bothersomeness}

Participants rated their pain bothersomeness during the previous 7 days on a $0-10$ numeric rating scale anchored by 0 (not at all bothersome) and 10 (extremely bothersome) that is commonly used in chronic low back pain research [58], "How bothersome has your pain been during the past week?"

\section{National Institutes of Health PROMIS Measures}

The National Institutes of Health PROMIS short-form measures have been applied in pain research [59-67], and selected domains were identified by the Initiative on Methods, Measurement, and Pain Assessment in Clinical Trials [68,69] as core outcomes. Respondents reference the previous 7 days to rate items for pain intensity (version 3a), sleep disturbance (version 6a), physical function (version 6b), depression (version 6a), anxiety (version 6a), and social isolation (4a). Higher scores on PROMIS measures indicate greater symptom severity, except for physical function, wherein higher scores reflect better function. The web-based PROMIS assessment center [70] software [71] was used to calculate the short-form $\mathrm{T}$ scores using Item Response Theory scoring algorithms that apply the Bayesian expected A posteriori method [72]. Depression, anxiety, and physical function are major correlates of chronic pain [73-76], and as such, changes in these variables were examined as a function of treatment. Social isolation was included because of the COVID-19 pandemic. We did not expect it to change, per se, but given the dynamic nature of COVID-19 and lockdowns, we measured this variable throughout the study.

\section{Sample Size and Statistical Analysis}

The planned enrollment accounted for $4 \%$ attrition in each study arm. The enrolled sample size ensured significant statistical power $(80 \%)$ to detect medium-to-large treatment effects between the two treatment groups. Of the 104 enrolled participants (ER: 50/104, 48.1\%; WLC: 54/104, 51.9\%), intent-to-treat analyses were used to analyze the data of 101 (97.1\%) participants (ER: 50/101, 49.5\%; WLC: 51/101, 50.5\%) who met the study criteria and completed at least one survey.

Baseline scores on continuous variables were summarized using mean (SD), and categorical variable scores were summarized by count and proportions. The differences between conditions 
at baseline for continuous and categorical variables for the treatment groups were assessed using one-way analysis of variance and Fisher exact test, respectively. For primary analyses, we used a between-within mixed design to assess the effect of treatment, time, and treatment $\times$ time, where time represented the within-subjects factor and treatment was specified as a between-subjects factor. Within-subjects' dependency was modeled by specifying participants as a random effect with a heterogeneous compound symmetry covariance structure. Similar analyses were used to examine the treatment effects for all secondary and tertiary outcomes at each posttreatment month. Intention-to-treat analysis was used to investigate the causal effects of the treatment. All statistical significances were applied at a two-sided level of 0.05 , with Benjamini-Hochberg adjustment for multiple comparisons. Cohen $d$ effect sizes for the between-subjects' factor were calculated from the between-subjects' $F$ statistic adjusting for sample size. Responder analyses were conducted by calculating the proportion of participants with 15\% (minimal), $30 \%$ (moderate), and 50\% (substantial) improvement from baseline for pain-related outcomes. All data processing and statistical analyses were performed using the SAS version 9.4 (SAS Institute, Inc).

\section{Results}

\section{Study Participants}

Figure 2 shows the CONSORT (Consolidated Standards of Reporting Trials) diagram for the study. In total, 196 individuals were assessed for initial eligibility, and 92 were excluded because they did not meet the study criteria. In total, 104 participants were enrolled and randomized, and 101 (97.1\%) participants completed the baseline surveys (ER: 50/101, 49.5\%; WLC: $51 / 101,50.5 \%$ ). A total of 49 participants received the ER intervention, of whom $2(4 \%)$ were lost to follow-up due to feeling unwell or being too busy to complete follow-up surveys. A final sample of 94 participants provided complete follow-up data. Nearly $94 \%$ (47/50) of participants in the ER group and $86 \%(44 / 51)$ of participants in the WLC group completed their 3-month follow-up surveys (end of study).

Figure 2. CONSORT (Consolidated Standards of Reporting Trials) flow diagram. ER: empowered relief; ITT: intention-to-treat; WLC: waitlist control.

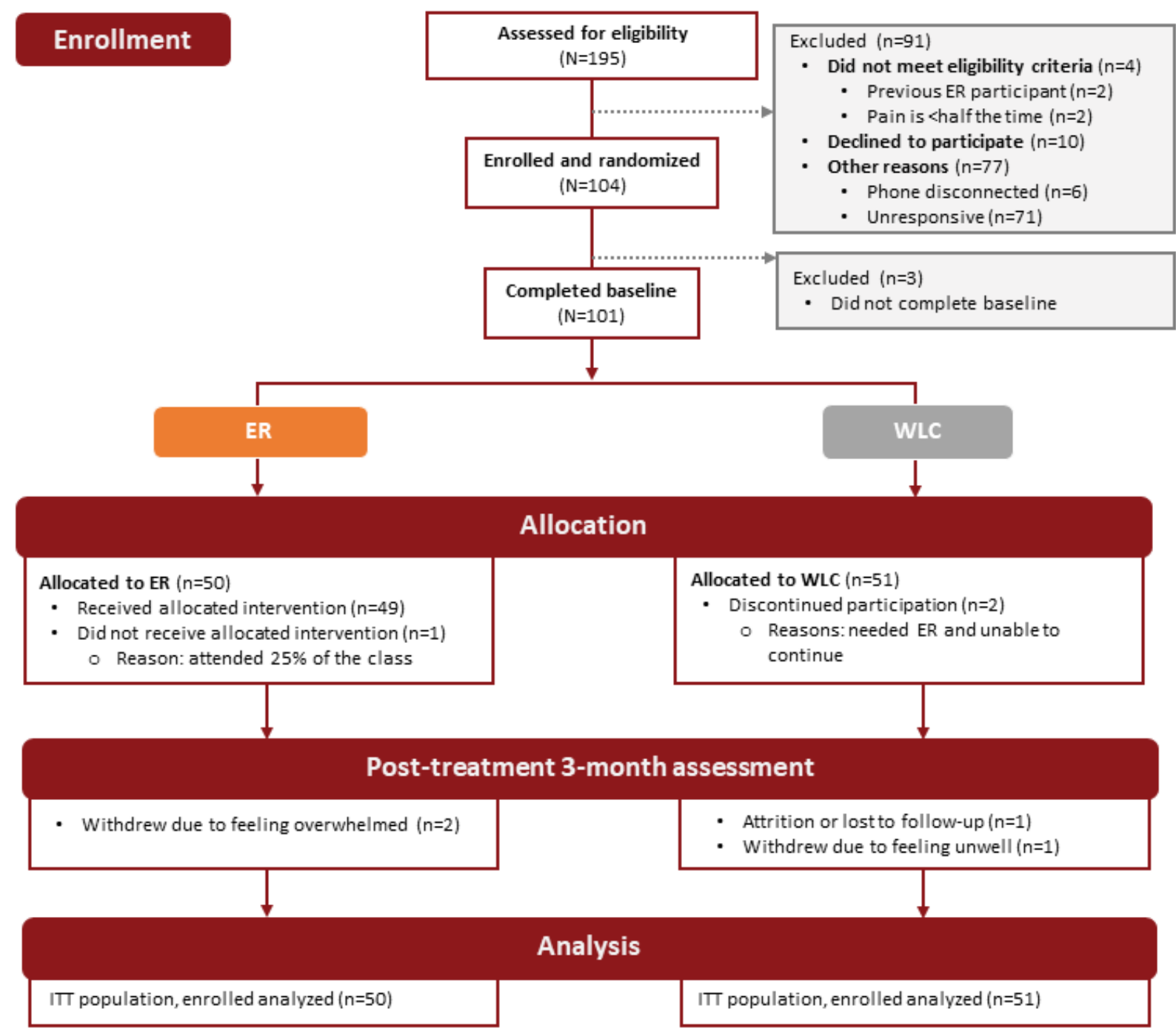


Table 2 shows the baseline demographic characteristics of the study arm. The sample included 101 participants recruited primarily from northern California. The sample was predominantly female $(70 / 101,69.3 \%)$, White $(75 / 101,74.3 \%)$, and with at least some college education $(66 / 101,65.3 \%)$, and a mean age of 49.76 years (SD 13.90; range 24.00-78.00). No significant between-group differences were observed for any demographic variables, demonstrating that randomization was effective. 
Table 2. Baseline demographic characteristics by treatment group.

\begin{tabular}{|c|c|c|c|}
\hline \multirow[t]{2}{*}{ Demographics } & \multicolumn{2}{|l|}{ Values } & \multirow[t]{2}{*}{$P$ value } \\
\hline & Treatment group with empowered relief $(n=50)$ & $\mathrm{WLC}^{\mathrm{a}}(\mathrm{n}=51)$ & \\
\hline Gender, n (\%) & & & $.15^{\mathrm{b}}$ \\
\hline Male & $12(24)$ & $19(37)$ & \\
\hline Female & $38(76)$ & $32(63)$ & \\
\hline Age (years) & & & $.34^{\mathrm{c}}$ \\
\hline Mean (SD; range) & $48.6(14.1 ; 26.0-78.0)$ & $50.9(13.7 ; 24.0-74.0)$ & \\
\hline Median (IQR) & $48.5(35.0-59.0)$ & $51.0(40.0-67.0)$ & \\
\hline Race, $\mathbf{n}(\%)$ & & & $.90^{\mathrm{b}}$ \\
\hline Asian & $6(12)$ & $7(14)$ & \\
\hline White & $37(74)$ & $38(75)$ & \\
\hline Black or African American & $1(2)$ & $0(0)$ & \\
\hline More than one race & $3(6)$ & $3(6)$ & \\
\hline Other or unknown & $3(6)$ & $3(6)$ & \\
\hline Education, n (\%) & & & $.20^{\mathrm{b}}$ \\
\hline High school graduate & $1(2)$ & $1(2)$ & \\
\hline Some college & $7(14)$ & $7(14)$ & \\
\hline Associate & $8(16)$ & $11(22)$ & \\
\hline Undergraduate & $14(28)$ & $19(37)$ & \\
\hline Master's degree & $13(26)$ & $10(20)$ & \\
\hline Professional degree & $1(2)$ & $3(6)$ & \\
\hline Doctoral degree & $6(12)$ & $0(0)$ & \\
\hline Employment, n (\%) & & & $.72^{\mathrm{b}}$ \\
\hline Part-time & $10(20)$ & $10(20)$ & \\
\hline Full-time & $15(30)$ & $18(35)$ & \\
\hline Full-time homemaker & $0(0)$ & $4(8)$ & \\
\hline Temporarily laid off & $3(6)$ & $1(2)$ & \\
\hline Unemployed & $3(6)$ & $2(4)$ & \\
\hline Looking for work, unemployed & $3(6)$ & $2(4)$ & \\
\hline Disabled or not working due to pain & $5(10)$ & $4(8)$ & \\
\hline Retired & $5(10)$ & $6(12)$ & \\
\hline Student, currently employed & $1(2)$ & $1(2)$ & \\
\hline Student, not currently employed & $1(2)$ & $0(0)$ & \\
\hline Other & $4(8)$ & $3(6)$ & \\
\hline Income (US \$), n (\%) & & & $.64^{\mathrm{b}}$ \\
\hline$<10,000$ & $1(2)$ & $2(4)$ & \\
\hline$>25,000$ & $4(8)$ & $7(14)$ & \\
\hline $25,000-44,999$ & $6(12)$ & $3(6)$ & \\
\hline $45,000-64,999$ & $2(4)$ & $3(6)$ & \\
\hline $65,000-84,999$ & $2(4)$ & $4(8)$ & \\
\hline $85,000-104,999$ & $7(14)$ & $3(6)$ & \\
\hline $105,000-124,999$ & $7(14)$ & $10(20)$ & \\
\hline
\end{tabular}




\begin{tabular}{lll}
\hline Demographics & Values & \\
& Treatment group with empowered relief $(\mathrm{n}=50)$ & WLC $^{\mathrm{a}}(\mathrm{n}=51)$ \\
\hline$>125,000$ & $15(30)$ & $14(27)$ \\
Missing & $6(12)$ & $5(10)$ \\
Relationship, $\mathbf{n}(\boldsymbol{\%})$ & & $9(18)$ \\
Never married & $10(20)$ & $26(51)$ \\
Married & $26(52)$ & $7(14)$ \\
Divorced & $6(12)$ & $2(4)$ \\
Separated & $1(2)$ & $2(4)$ \\
Widowed & $0(0)$ & $4(8)$ \\
Partnered and living together & $5(10)$ & $1(2)$ \\
In a relationship but not living together & $2(4)$ & \\
\hline
\end{tabular}

${ }^{\mathrm{a}}$ WLC: waitlist control.

${ }^{\mathrm{b}}$ Chi-square $P$ value.

${ }^{\mathrm{c}}$ Kruskal-Wallis $P$ value.

Table 3 presents the baseline pain and clinical characteristics of the sample by the study arm. The mean pain intensity $\mathrm{T}$ score was 62.2 (mean 50, SD 10). No significant differences were observed between the two study groups for any of the variables assessed, except for sleep disturbance $(P=.04)$, which was controlled for in the analysis. 
Table 3. Baseline clinical variables by treatment group.

\begin{tabular}{|c|c|c|c|}
\hline \multirow[t]{2}{*}{ Variables } & \multicolumn{2}{|l|}{ Values } & \multirow[t]{2}{*}{$P$ value ${ }^{\mathrm{b}}$} \\
\hline & Treatment group with empowered relief $(\mathrm{n}=50)$ & $\mathrm{WLC}^{\mathrm{a}}(\mathrm{n}=51)$ & \\
\hline Pain catastrophizing & & & .86 \\
\hline Mean (SD; range) & $20.6(10.1 ; 2.0-47.0)$ & $20.2(10.7 ; 2.0-43.0)$ & \\
\hline Median (IQR) & $19.0(13.0-28.0)$ & $19.0(12.0-29.0)$ & \\
\hline Average pain intensity & & & .60 \\
\hline Mean (SD; range) & $62.2(5.7 ; 48.0-81.9)$ & $62.5(7.7 ; 36.3-81.9)$ & \\
\hline Median (IQR) & $62.6(58.9-64.1)$ & $62.6(60.2-67.8)$ & \\
\hline Pain bothersomeness & & & .28 \\
\hline Mean (SD; range) & $5.8(2.0 ; 0.0-10.0)$ & $6.3(2.0 ; 2.0-10.0)$ & \\
\hline Median (IQR) & $6.0(4.0-7.0)$ & $6.0(5.0-7.0)$ & \\
\hline \multicolumn{4}{|l|}{ PROMIS $^{\mathrm{c}}$} \\
\hline Depression & & & .95 \\
\hline Mean (SD; range) & $57.3(9.6 ; 38.4-73.0)$ & $57.8(9.3 ; 38.4-80.2)$ & \\
\hline Median (IQR) & $58.2(50.6-65.4)$ & $56.4(52.3-64.7)$ & \\
\hline Anxiety & & & .36 \\
\hline Mean (SD; range) & $59.6(8.3 ; 39.1-82.4)$ & $60.4(8.9 ; 39.1-79.5)$ & \\
\hline Median (IQR) & $60.7(52.9-64.5)$ & $61.8(54.4-66.1)$ & \\
\hline Physical function & & & .99 \\
\hline Mean (SD; range) & $40.8(6.3 ; 28.7-59.0)$ & $41.1(7.2 ; 24.8-59.0)$ & \\
\hline Median (IQR) & $40.4(36.0-45.1)$ & $40.5(36.7-44.1)$ & \\
\hline Interference & & & .82 \\
\hline Mean (SD; range) & $59.7(6.6 ; 41.1-76.2)$ & $60.3(5.4 ; 51.0-76.2)$ & \\
\hline Median (IQR) & $59.5(55.7-64.8)$ & $60.0(56.2-63.8)$ & \\
\hline Fatigue & & & .79 \\
\hline Mean (SD; range) & $61.6(8.4 ; 33.7-75.8)$ & $62.4(9.2 ; 33.7-75.8)$ & \\
\hline Median (IQR) & $62.8(57.1-66.8)$ & $62.7(57.1-68.8)$ & \\
\hline Social isolation & & & .23 \\
\hline Mean (SD; range) & $53.5(11.0 ; 34.8-74.2)$ & $52.3(9.4 ; 34.8-74.2)$ & \\
\hline Median (IQR) & $56.2(47.9-62.1)$ & $51.7(47.9-56.2)$ & \\
\hline Anger & & & .23 \\
\hline Mean (SD; range) & $54.6(9.1 ; 32.9-82.9)$ & $55.5(12.0 ; 32.9-77.1)$ & \\
\hline Median (IQR) & $54.7(47.9-60.7)$ & $57.8(49.8-63.8)$ & \\
\hline Sleep disturbance & & & .04 \\
\hline Mean (SD; range) & $56.3(9.0 ; 36.4-76.1)$ & $59.5(8.5 ; 36.6-76.1)$ & \\
\hline Median (IQR) & $54.5(51.3-61.8)$ & $60.0(53.9-64.7)$ & \\
\hline
\end{tabular}

${ }^{\mathrm{a}} \mathrm{WLC}$ : waitlist control.

${ }^{\mathrm{b}}$ Kruskal-Wallis $P$ value

${ }^{\mathrm{c}}$ PROMIS: Patient-Reported Outcomes Measurement Information System.

\section{Treatment Satisfaction (ER Group Only)}

For satisfaction with ER treatment on a scale of 0 (completely dissatisfied) to 10 (completely satisfied), participants $(\mathrm{n}=47)$ reported high satisfaction with all items related to overall satisfaction with the class (mean 8.26, SD 1.57), likelihood of recommending the class (mean 8.77, SD 1.43), class relevance (mean 7.94, SD 2.03), the usefulness of the presented 
information (mean 8.37, SD 1.79), and likelihood of using the skills and information learned (mean 8.66, SD 1.58).

\section{Primary Outcomes}

Table 4 reports the between-group comparisons at 2 weeks and 1-3 months posttreatment for all outcomes. Figures 3-12 compare group effects over time for pain-related outcomes. For all figures, the trend of the pain-related variables is displayed over time for participants in both groups. Values in the $\mathrm{x}$-axis refer to the number of weeks in the study, where 0 represents the baseline (roughly 4 days preintervention) time-point. The color band represents the $95 \% \mathrm{CI}$ for the mean outcome variable. Overlapping bands indicate nonsignificant treatment group differences ( $P$ value) of simple main effects within each time-point. The corresponding model effects for each outcome are displayed in Figures 3-12.

We observed a significant treatment effect $(P=.005)$ on pain catastrophizing; on average, the ER group had lower PCS scores than the WLC group (Cohen $d=0.50$ ). Separately, we observed a time effect; the average PCS significantly decreased over time for both study groups (time effect; $P<.001$ ). Most importantly, the decrease was greater for ER versus WLC (groupxtime effect; $P<.001)$. At 3-month posttreatment, clinically meaningful reductions in PCS were found for ER but not for WLC (ER: PCS $-8.72,42.25 \%$ reduction; WLC: PCS $-2.25,11.13 \%$ reduction). Figure 3 displays the average PCS at 3 months by study arm, and Table 4 displays the between-group comparisons for PCS at baseline and posttreatment months. ER was superior to WLC (difference $-6.05,95 \%$ CI -9.92 to $-2.18 ; P=.002$ at 3-month posttreatment.

The ER group effect size was 0.89 , with combined results showing a large effect size and moderate clinical importance. As much as $62 \%(31 / 50)$ of ER and 24\% (12/51) of WLC participants achieved a $30 \%$ or more reduction in PCS. For ER, $46 \%(23 / 50)$ achieved $\geq 50 \%$ in PCS reduction, whereas for WLC, $12 \%(6 / 51)$ reached that threshold. 
Table 4. Outcome measures from baseline to 3 months by treatment group with between-group comparisons.

\begin{tabular}{llll}
\hline Outcome measure time-point & $\begin{array}{l}\text { Empowered relief, } \\
\text { mean (SE) }\end{array}$ & $\begin{array}{l}\text { WLC, }{ }^{\mathrm{a}} \text { mean } \\
(\mathrm{SE})\end{array}$ & $\begin{array}{l}\text { Between-group differences } \\
(\mathrm{Cohen} d)\end{array}$ \\
\hline
\end{tabular}

\section{Pain catastrophizing}

Baseline

2 weeks posttreatment

1 month posttreatment

2 months posttreatment

3 months posttreatment

\section{Pain bothersomeness}

Baseline

2 weeks posttreatment

1 month posttreatment

2 months posttreatment

3 months posttreatment

\section{PROMIS $^{\mathrm{c}}$}

\section{Pain intensity}

Baseline

2 weeks posttreatment

1 month posttreatment

2 months posttreatment

3 months posttreatment

\section{Physical function}

Baseline

2 weeks posttreatment

1 month posttreatment

2 months posttreatment

3 months posttreatment

\section{Pain interference}

Baseline

2 weeks posttreatment

1 month posttreatment

2 months posttreatment

3 months posttreatment

\section{Anxiety}

Baseline

2 weeks posttreatment

1 month posttreatment

2 months posttreatment

3 months posttreatment

\section{Depression}

Baseline

2 weeks posttreatment

$20.64(1.53)$
$13.85(1.37)$
$12.13(1.37)$
$11.16(1.3)$
$11.92(1.39)$

$5.78(0.29)$

$5.13(0.33)$

$4.81(0.32)$

$4.71(0.34)$

$4.62(0.35)$

$62.24(0.98)$

$59.81(0.98)$

$57.96(0.95)$

$59.17(1)$

$57.26(1.18)$

$40.76(0.94)$

$41.65(0.91)$

$42.13(0.93)$

$42.18(0.94)$

$42.55(1.15)$

$59.71(0.86)$

$58.4(0.92)$

$57.56(0.89)$

$57.47(0.93)$

$57.51(1.12)$

$59.57(1.23)$

$58.12(1.33)$

$56.06(1.25)$

$56.18(1.28)$

54.85 (1.28)

$20.22(1.52)$
$19.52(1.38)$
$18.7(1.35)$
$18.5(1.31)$
$17.97(1.39)$

$0.42(2.16 ;-3.81$ to 4.66$)$

.84

0.04

$-5.67(1.94 ;-9.49$ to -1.85$)$

.004

$<.001$

0.57

$-6.57(1.93 ;-10.36$ to -2.78$)$

$-7.34(1.85 ;-10.97$ to -3.71$)$

$<.001$

$-6.05(1.97 ;-9.92$ to -2.18$)$

.002

0.62

0.76

0.64

$6.3(0.29)$

$-0.52(0.40 ;-1.31$ to 0.27$)$

.20

0.26

$5.66(0.34)$

$-0.53(0.47 ;-1.46$ to 0.4$)$

.26

0.27

$5.92(0.31)$

$-1.10(0.44 ;-1.98$ to -0.23$)$

.01

0.57

$5.84(0.34)$

$-1.13(0.48 ;-2.07$ to -0.19$)$

0.60

$5.92(0.36)$

$-1.30(0.50 ;-2.29$ to -0.31$)$

\section{$62.53(0.97)$}

$61.61(0.99)$

$62.06(0.94)$

62.55 (1.01)

61.07 (1.2)

$41.12(0.94)$

$40.42(0.92)$

40.77 (0.93)

39.97 (0.95)

$41.27(1.16)$

$60.27(0.86)$

$60.41(0.93)$

$60.02(0.88)$

$59.79(0.94)$

$59.51(1.14)$

$60.41(1.22)$

60.4 (1.33)

$59.79(1.23)$

$59.98(1.28)$

59.99 (1.28)
$-0.28(1.39 ;-3.01$ to 2.44$)$

$-1.8(1.40 ;-4.54$ to 0.94$)$

$-4.1(1.34 ;-6.73$ to -1.47$)$

$-3.38(1.42 ;-6.17$ to -0.59$)$

$-3.81(1.68 ;-7.12$ to -0.49$)$

$-0.37(1.33 ;-2.98$ to 2.24$)$

$1.23(1.29 ;-1.31$ to 3.77$)$

$1.35(1.31 ;-1.23$ to 3.94$)$

$2.22(1.33 ;-0.41$ to 4.84$)$

$1.28(1.63 ;-1.93$ to 4.5$)$

$-0.56(1.22 ;-2.96$ to 1.84$)$

$-2(1.31 ;-4.59$ to 0.58$)$

$-2.46(1.25 ;-4.92$ to 0$)$

$-2.32(1.33 ;-4.94$ to 0.29$)$

$-1.99(1.60 ;-5.14$ to 1.15$)$

$-0.84(1.73 ;-4.24$ to 2.56$)$

.63

$-2.28(1.88 ;-5.97$ to 1.42$)$

.23

.03

$-3.72(1.75 ;-7.17$ to -0.28$)$

$-3.8(1.81 ;-7.36$ to -0.25$)$

.04

$-5.14(1.81 ;-8.7$ to -1.58$)$
0.04

0.27

0.62

0.54

0.60

0.05

0.24

0.26

0.41

0.25

0.09

0.32

0.43

0.43

0.33

0.10

0.28

0.41

0.49

0.68 


\begin{tabular}{|c|c|c|c|c|c|}
\hline \multirow[t]{2}{*}{ Outcome measure time-point } & \multirow{2}{*}{$\begin{array}{l}\text { Empowered relief, } \\
\text { mean (SE) }\end{array}$} & \multirow{2}{*}{$\begin{array}{l}\text { WLC, }{ }^{\mathrm{a}} \text { mean } \\
(\mathrm{SE})\end{array}$} & \multicolumn{2}{|l|}{ Between-group differences } & \multirow{2}{*}{$\begin{array}{l}\text { Effect size } \\
(\text { Cohen } d)\end{array}$} \\
\hline & & & Mean difference (SE; 95\% CI) & $P$ value & \\
\hline 1 month posttreatment & $53.58(1.25)$ & $57.24(1.24)$ & $-3.65(1.76 ;-7.11$ to -0.19$)$ & .04 & 0.37 \\
\hline 2 months posttreatment & $53.5(1.21)$ & $57.55(1.21)$ & $-4.05(1.71 ;-7.41$ to -0.7$)$ & .02 & 0.45 \\
\hline 3 months posttreatment & $53.89(1.29)$ & $57.26(1.29)$ & $-3.37(1.82 ;-6.95$ to 0.21$)$ & .06 & 0.40 \\
\hline \multicolumn{6}{|l|}{ Social isolation } \\
\hline Baseline & $53.49(1.47)$ & $52.34(1.47)$ & $1.15(2.08 ;-2.95$ to 5.25$)$ & .58 & 0.11 \\
\hline 2 weeks posttreatment & $51.59(1.45)$ & $52.75(1.46)$ & $-1.16(2.06 ;-5.21$ to 2.89$)$ & .57 & 0.19 \\
\hline 1 month posttreatment & $51.06(1.35)$ & $52.48(1.34)$ & $-1.42(1.90 ;-5.16$ to 2.32$)$ & .46 & 0.23 \\
\hline 2 months posttreatment & $50.12(1.48)$ & $52.49(1.49)$ & $-2.37(2.10 ;-6.5$ to 1.75$)$ & .26 & 0.33 \\
\hline 3 months posttreatment & $49.5(1.42)$ & $52.37(1.43)$ & $-2.87(2.02 ;-6.84$ to 1.1$)$ & .16 & 0.38 \\
\hline \multicolumn{6}{|l|}{ Anger } \\
\hline Baseline & $54.64(1.57)$ & $55.48(1.57)$ & $-0.84(2.22 ;-5.21$ to 3.53$)$ & .70 & 0.08 \\
\hline 2 weeks posttreatment & $52.84(1.45)$ & $56.46(1.47)$ & $-3.62(2.07 ;-7.68$ to 0.45$)$ & .08 & 0.34 \\
\hline 1 month posttreatment & $52.59(1.28)$ & $56.24(1.27)$ & $-3.65(1.8 ;-7.18$ to -0.11$)$ & .04 & 0.35 \\
\hline 2 months posttreatment & $50.88(1.37)$ & $55.2(1.39)$ & $-4.31(1.95 ;-8.15$ to -0.48$)$ & .03 & 0.44 \\
\hline 3 months posttreatment & $50.54(1.33)$ & $56(1.35)$ & $-5.47(1.89 ;-9.19$ to -1.75$)$ & .004 & 0.59 \\
\hline
\end{tabular}

${ }^{\mathrm{a}}$ WLC: waitlist control.

${ }^{\mathrm{b}}$ These are between-subjects effect size within each time frame.

${ }^{c}$ PROMIS: Patient-Reported Outcomes Measurement Information System.

Figure 3. Pain catastrophizing over time.

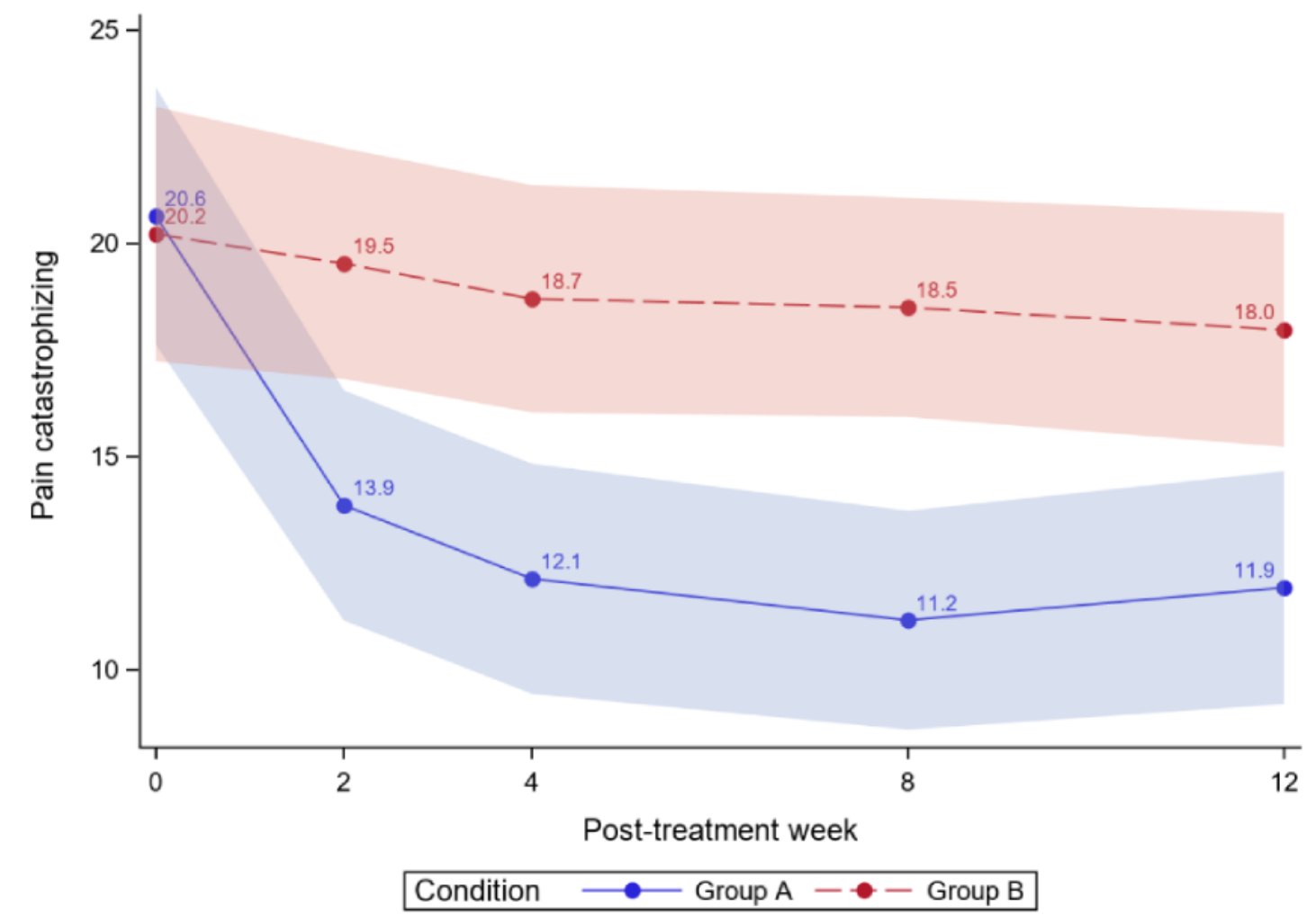


Figure 4. Pain intensity over time.

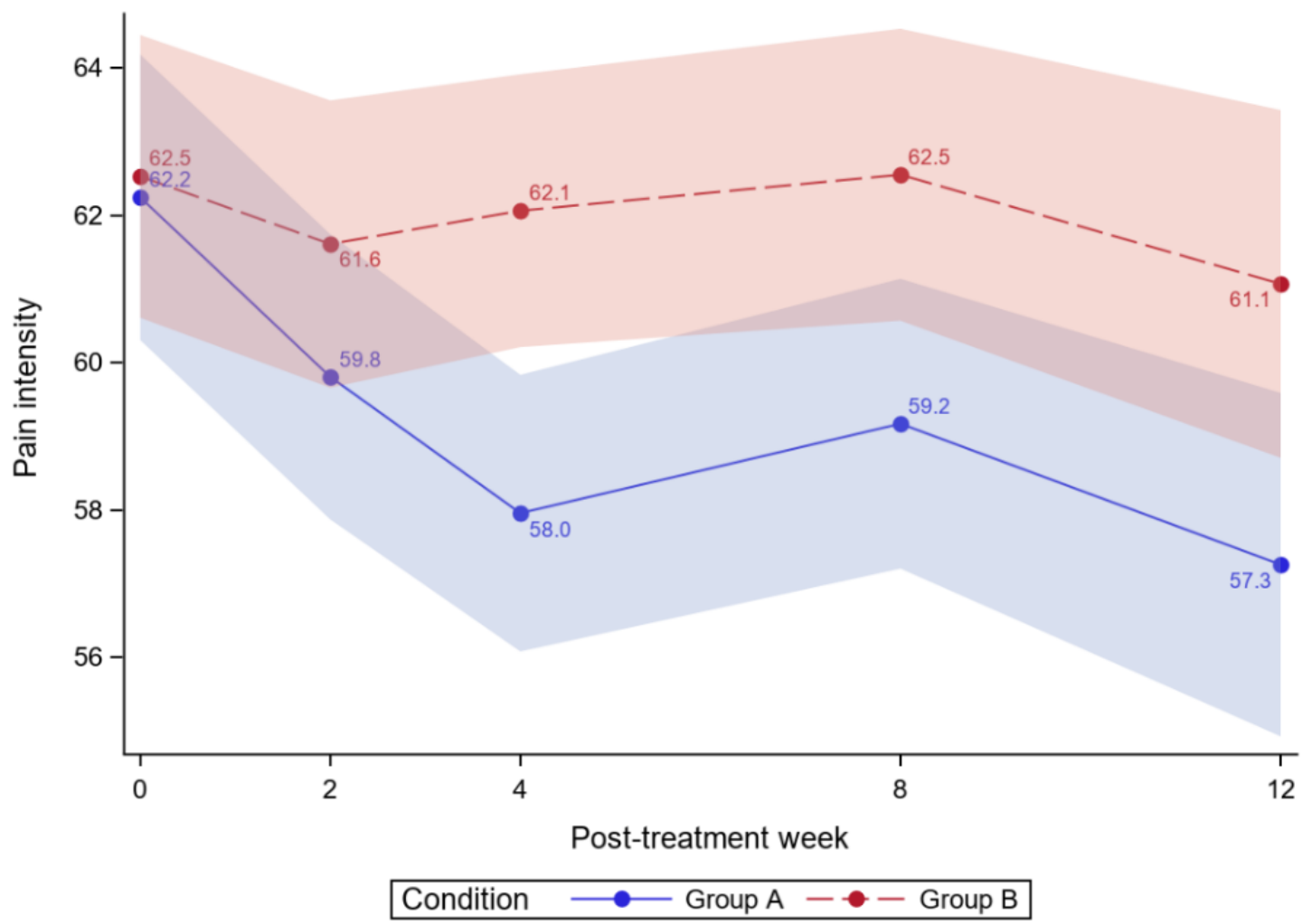

Figure 5. Pain bothersomeness over time.

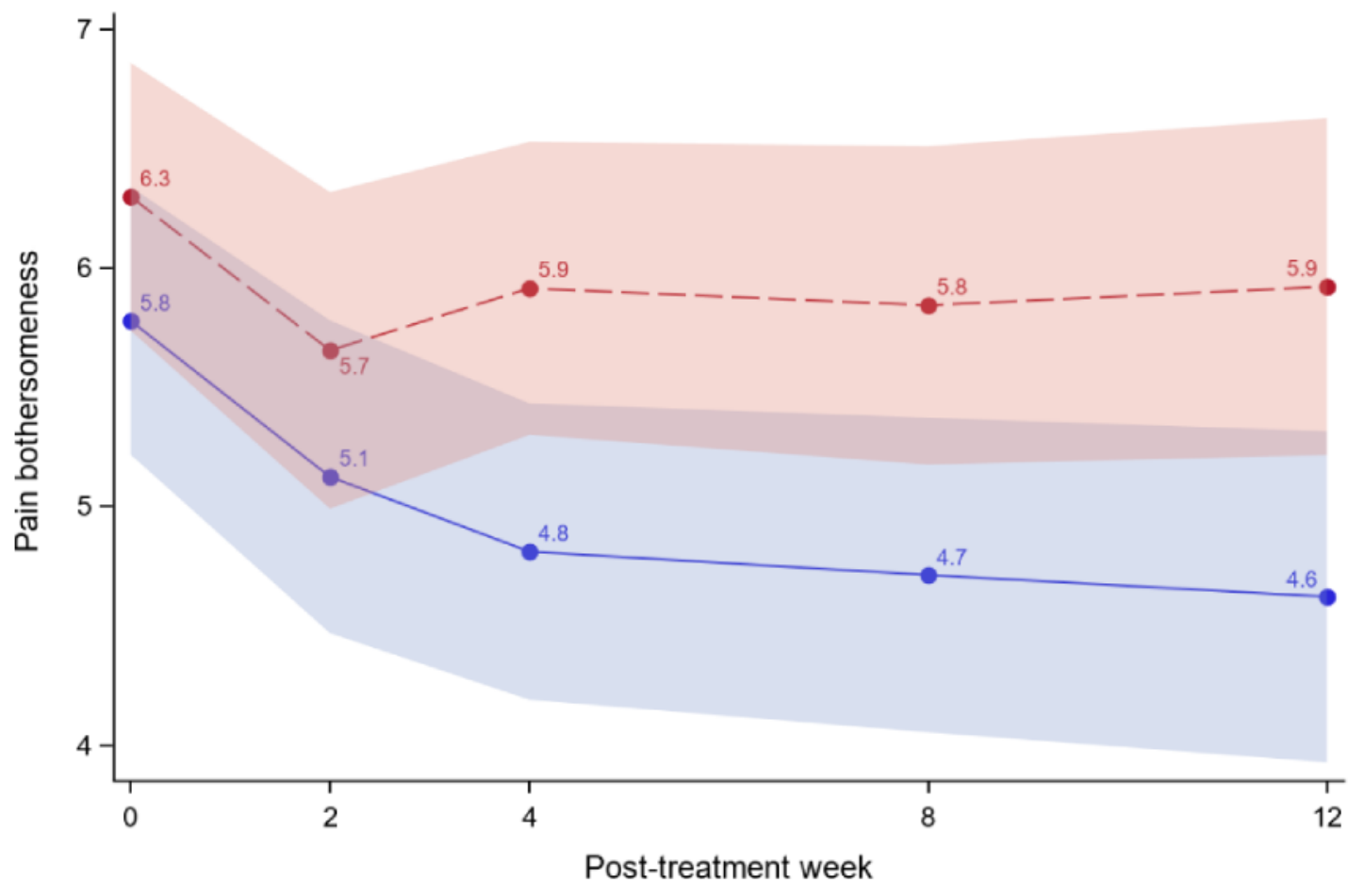

Condition $\longrightarrow$ Group A $\longrightarrow-$ Group B 
Figure 6. Sleep disturbance over time. PROMIS: Patient-Reported Outcomes Measurement Information System.

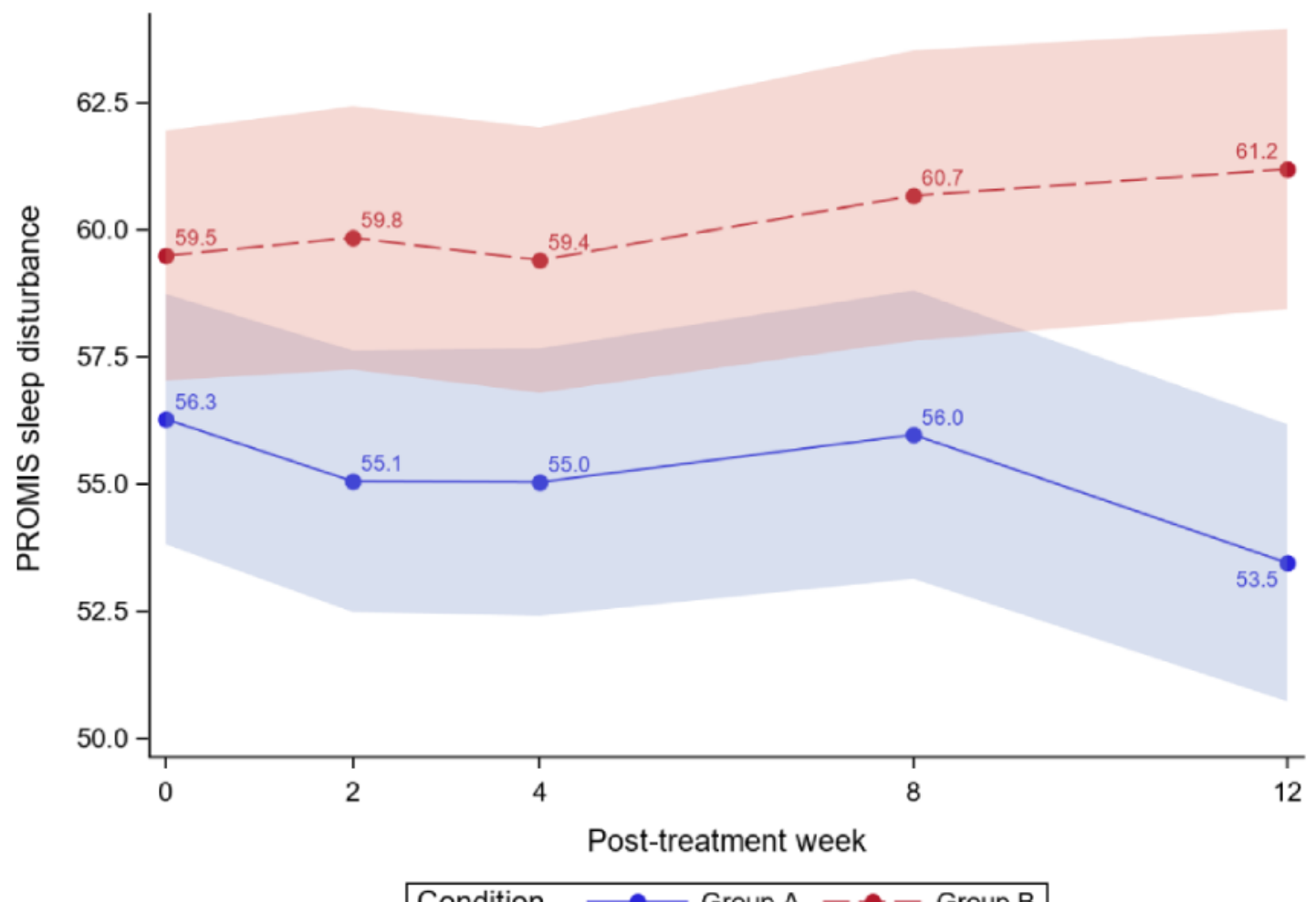

Figure 7. Anxiety over time. PROMIS: Patient-Reported Outcomes Measurement Information System.

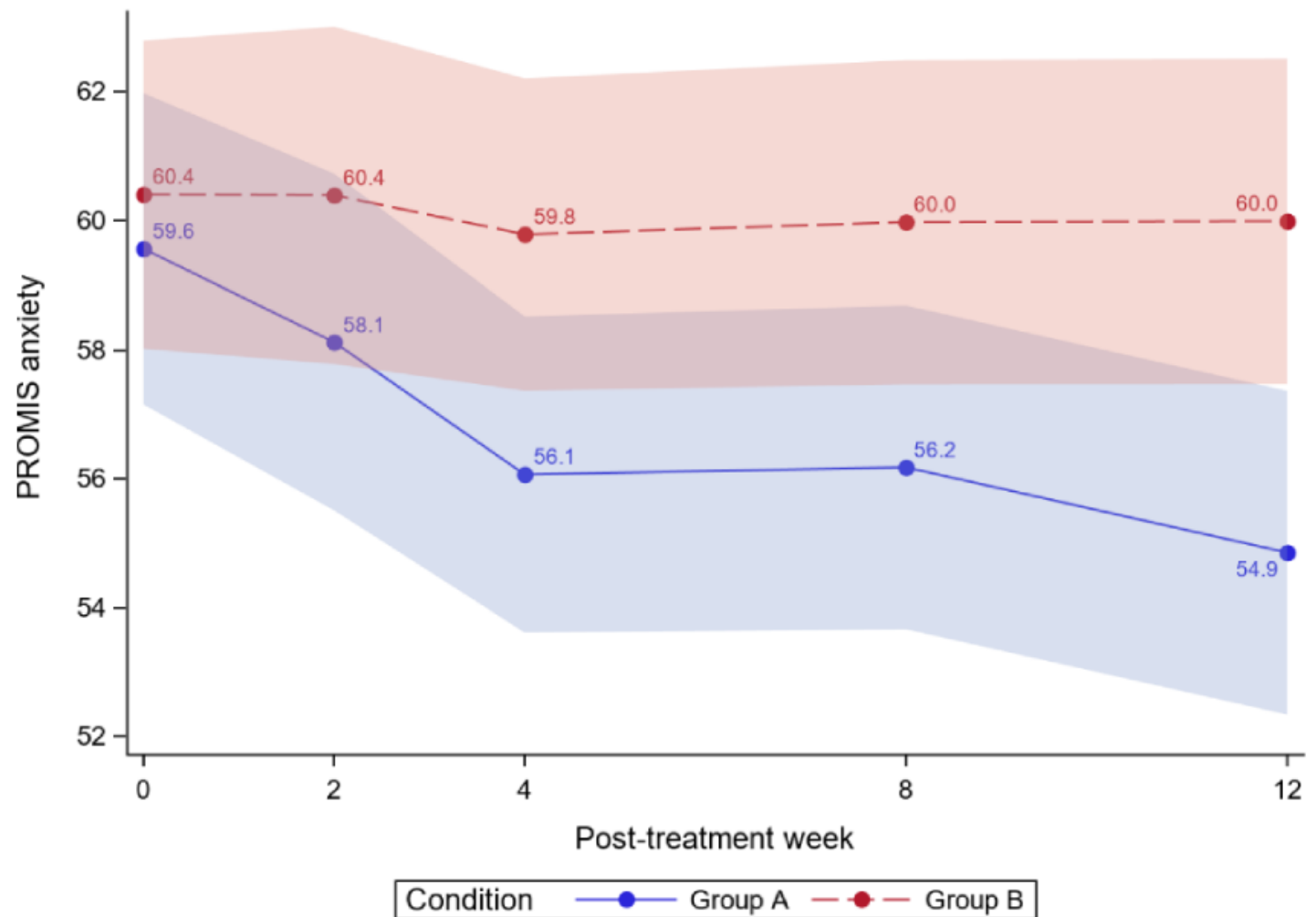


Figure 8. Depression over time. PROMIS: Patient-Reported Outcomes Measurement Information System.

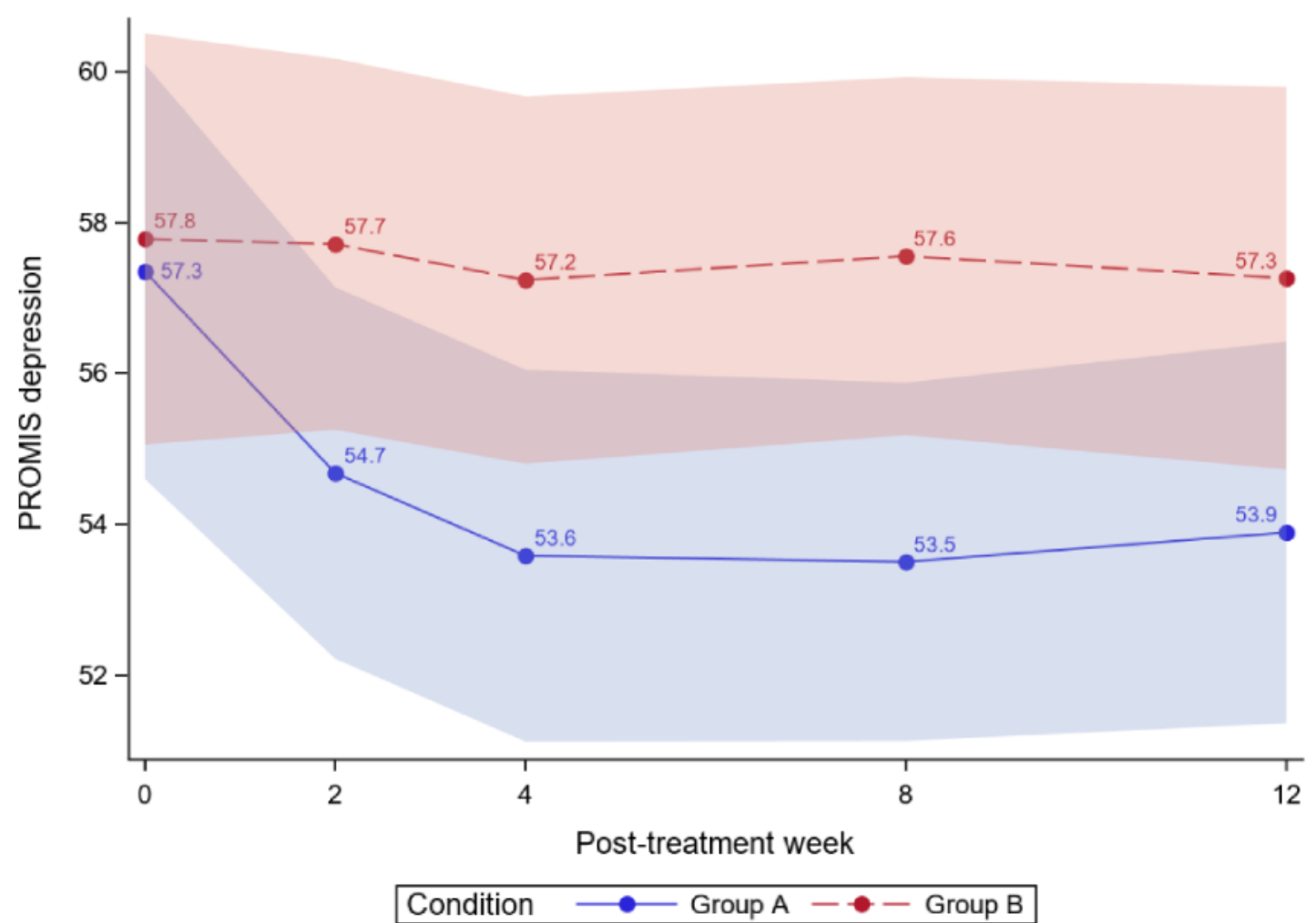

Figure 9. Physical function over time. PROMIS: Patient-Reported Outcomes Measurement Information System.

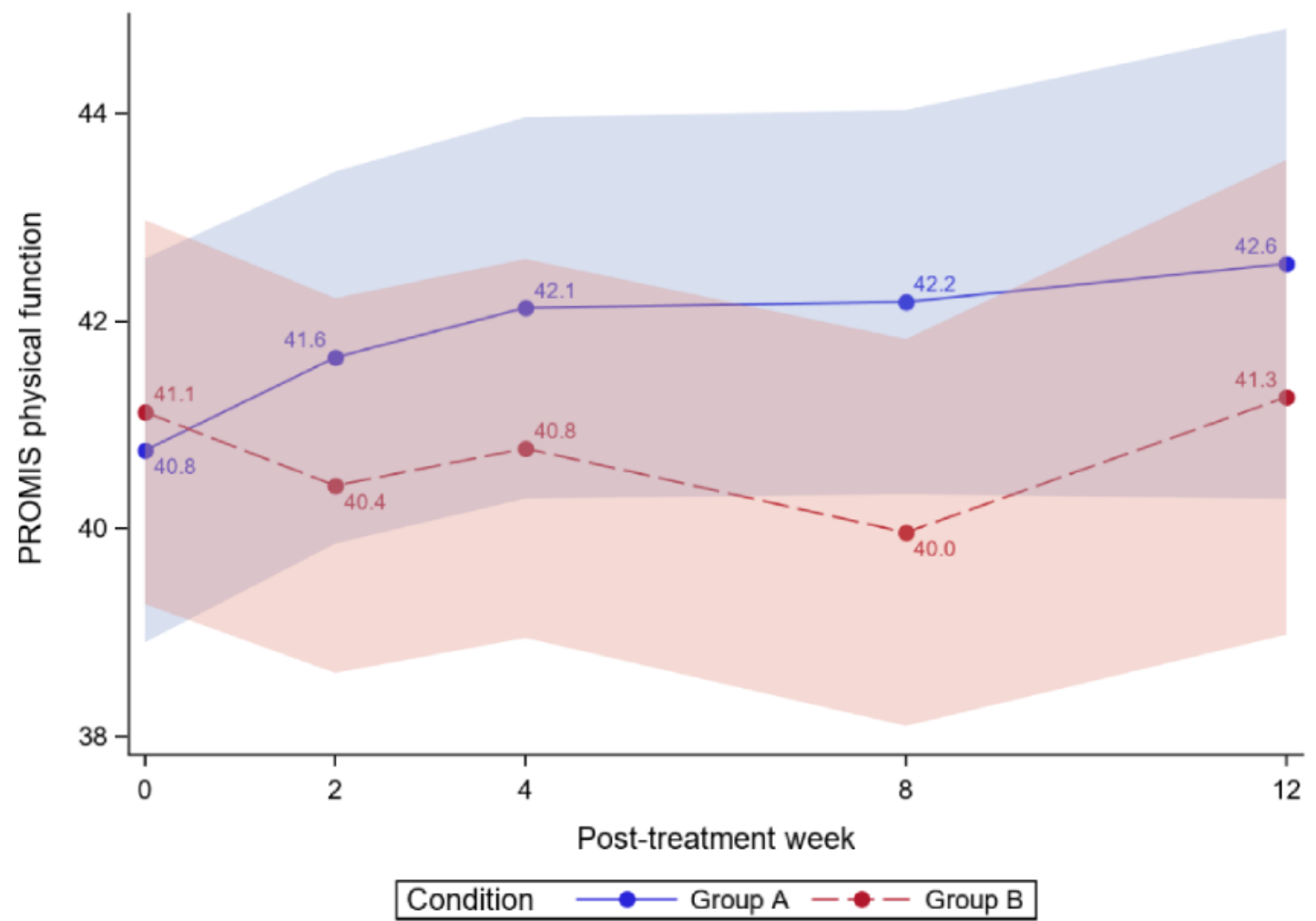


Figure 10. Pain interference over time. PROMIS: Patient-Reported Outcomes Measurement Information System.

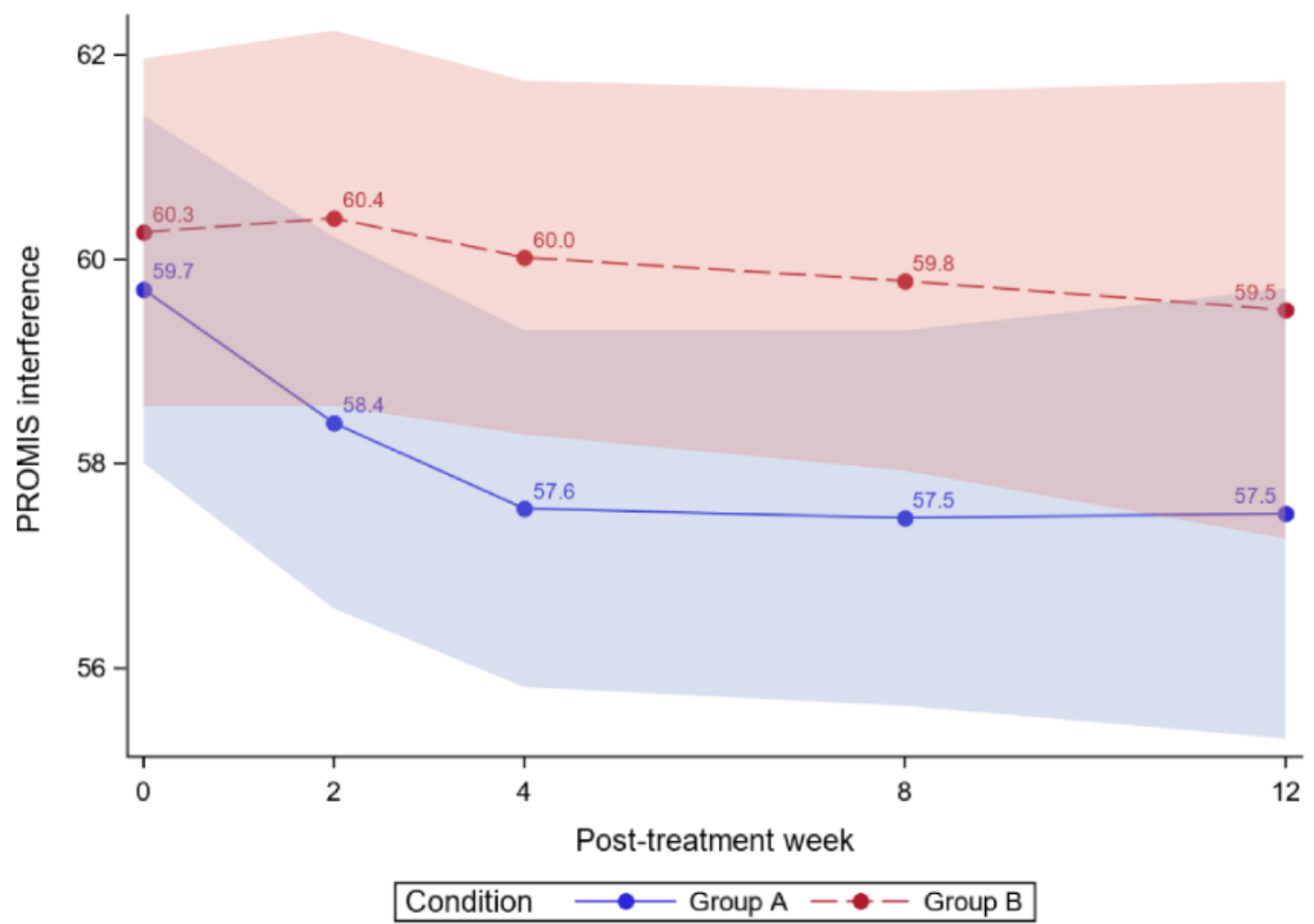

Figure 11. Social isolation over time. PROMIS: Patient-Reported Outcomes Measurement Information System.

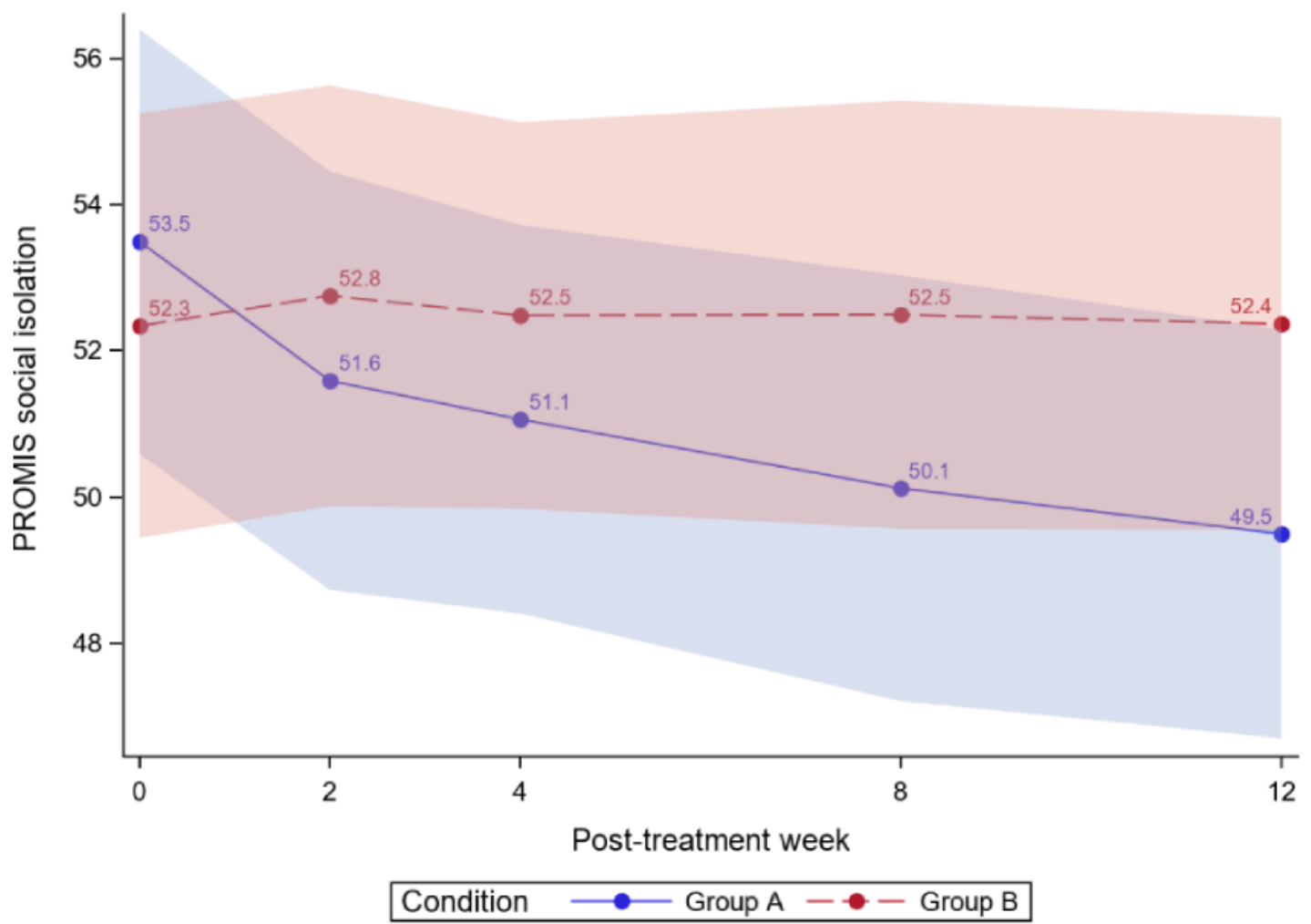


Figure 12. Anger over time. PROMIS: Patient-Reported Outcomes Measurement Information System.

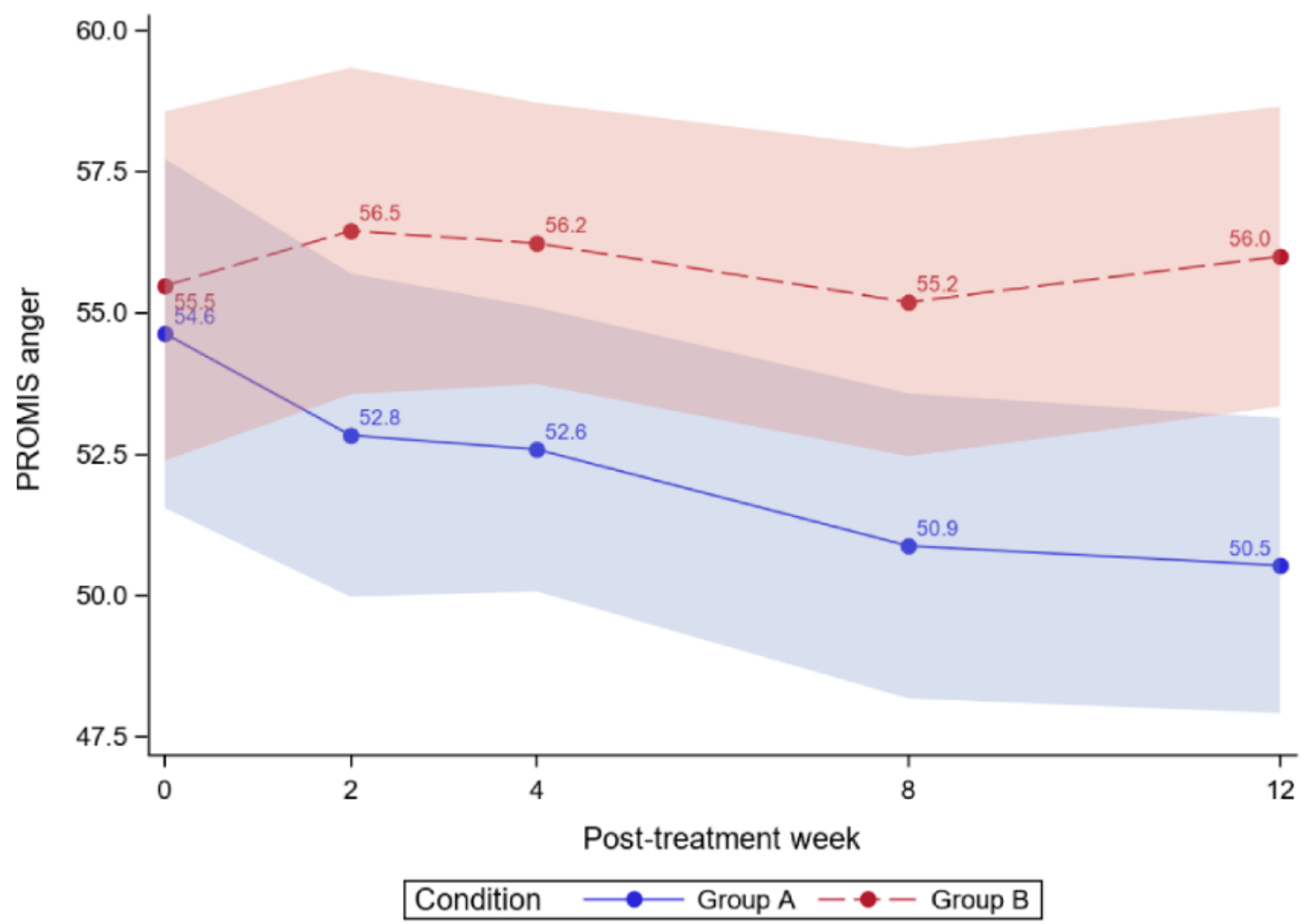

\section{Secondary Outcomes}

\section{Pain Intensity}

Analyzing all data from baseline to 3 months, we observed a significant treatment effect $(P=.02)$ on pain intensity; on average, the ER group had lower pain intensity scores than the WLC group (Cohen $d=0.41$ ). Separately, we observed a time effect; the average PCS significantly decreased over time for both study groups (time effect; $P<.001$ ). Most importantly, the decrease was greater for ER versus WLC (group $\times$ time effect; $P=.01)$.

At 3 months, significant reductions in pain intensity were found for ER but not for WLC (ER: pain intensity $-4.98,8 \%$ reduction; WLC: pain intensity $-1.46,2.34 \%$ reduction). Figure 4 displays the average pain intensity during the 3-month follow-up by the study arm, and Table 4 displays the between-group comparisons for pain intensity at baseline and posttreatment months. At 3 months, ER was superior to WLC (difference -6.05, 95\% CI -9.92 to $-2.18 ; P<.001)$. Notably, when applying the $15 \%$ threshold for clinical meaningfulness, the reduction in pain intensity was not clinically meaningful for either group.

The ER group effect size was 0.76 , with combined results showing a moderate-to-large effect size but no clinical importance. As much as 30\% (15/50) of ER and 6\% (3/51) of WLC participants achieved a $15 \%$ or greater reduction in pain intensity. For ER, 4\% (2/50) achieved $\geq 30 \%$ reduction in pain intensity, whereas for WLC, $2 \%(1 / 51)$ reached that threshold.

\section{Pain Bothersomeness}

We observed a time effect; average pain bothersomeness significantly decreased over time for both study groups (time effect; $P<.001)$; however, during the 1- to 3-month follow-ups, the between-group difference was significant $(P=.01$ to $P=.02)$ per Table 4 . This significance is largely driven by the initial decline in pain bothersomeness, which was steeper in the ER group and eventually plateaued for both groups (Figure 5). At 3 months, ER was superior to WLC (difference $-1.30,95 \%$ CI -2.29 to $-0.31 ; P=.01)$.

At 3 months, clinically meaningful reductions in pain bothersomeness were found for ER but not for WLC (ER: pain bothersomeness $-1.16,20.07 \%$ reduction; WLC: pain bothersomeness $-0.38,6.03 \%$ reduction). This meets the threshold for moderate clinical meaningfulness. Figure 6 displays the average pain bothersomeness during the 3-month follow-up by the study arm, and Table 4 displays the between-group comparisons for pain intensity at baseline and posttreatment months.

The ER group effect size was 0.61 , with combined results showing a moderate effect size and minimal clinical importance. As much as 34\% (17/50) of ER and 12\% (6/51) of WLC participants achieved $\geq 30 \%$ reduction in pain bothersomeness. For ER, $22 \%(11 / 50)$ achieved $\geq 50 \%$ in pain bothersomeness reduction, whereas for WLC, 3.9\% (2/51) reached that threshold.

\section{Sleep Disturbance}

At 3 months, significant reductions in sleep disturbance were found for ER but not for WLC (ER: 2.82, 5.01\% reduction; WLC: $1.7,2.86 \%$ increase; Table 4). Separately, we did not observe a time effect $(P=.52)$, and we observed a significant interaction effect $(P=.02)$. Sleep disturbance was lower in the ER group than in the WLC group $(P=.02)$. At 3 months, ER was superior to WLC (difference $-7.74,95 \% \mathrm{CI}-11.61$ to 
-3.87; $P<.001)$. The ER group effect size was 0.37 , with combined results showing a small-to-moderate effect size and no clinical importance. As much as $12 \%(6 / 50)$ of ER and $8 \%$ (4/51) of WLC participants achieved a $15 \%$ or more reduction in sleep disturbance.

The Benjamini-Hochberg adjustment for multiple comparisons was applied across primary and secondary variables, and statistical significance was maintained $(P=.02$ to $P<.001)$.

\section{Tertiary Outcomes}

\section{Anxiety}

At 3 months, significant reductions in anxiety were found for ER but not for WLC (ER: $-4.71,7.91 \%$ reduction; WLC: -0.42 , $0.7 \%$ reduction; Table 4$)$. Anxiety decreased over time in both groups, and the decline was significantly steeper in the ER group $(P=.009)$. ER was superior to WLC (difference $-5.14,95 \%$ CI -8.7 to $-1.58 ; P<.001)$. The ER group effect size was 0.59 , with combined results showing a moderate effect size and no clinical importance.

\section{Depression}

We did not observe a treatment effect $(P=.08)$ on depression. Separately, we observed a time effect; average depression significantly decreased over time for both study groups (time effect; $P=.002$ ). Most importantly, the decrease was greater for ER versus WLC (groupxtime effect; $P=.03$ ). However, between-group differences showed that ER was superior to WLC only during months 1 and $2(P=.04$ and $P=.02$, respectively). The overall interaction effect appears primarily driven by these two time-points and maintains the trend and directionality, but not statistically significant, at the 3-month follow-up $(P=.07)$.

\section{Physical Function}

We did not observe a treatment effect $(P=.36)$ or a time effect $(P=.36)$ on physical function. We observed a trend for the interaction effect $(P=.09)$. Between-subjects analysis revealed no significant differences at any of the follow-up time-points $(P=.30$ to $P=.43)$, except at 2 -months posttreatment $(P=.01)$.

\section{Exploratory Outcomes}

For social isolation, we observed a time effect; average social isolation scores significantly decreased over time for both study groups (time effect; $P=.02$ ). Most importantly, this was qualified by a significant group $x$ time interaction $(P=.02)$; there was a decrease in social isolation in the ER group but not in the WLC group. No time or interaction effects were observed for pain interference or anger.

\section{Zoom Platform Satisfaction}

Participants in the ER arm provided feedback on their experience using the Zoom platform on a scale from 1 (strongly disagree) to 7 (strongly agree), with higher scores indicating a higher endorsement of each item. Overall, participants reported high satisfaction with the platform, including the ease of operating Zoom (46/50, 92\% satisfaction: mean 6.45, SD 1.43), engaging with class material (mean 6.02, SD 1.45), comfort in engaging with Zoom instructor and participants (mean 5.68, SD 1.45), and feeling connected to the instructor (mean 5.43, SD 1.64).

\section{Discussion}

\section{Principal Findings}

We conducted the first Zoom-delivered randomized controlled trial of the group-based, SSI ER in a sample of individuals with mixed-etiology chronic pain. The primary goal of this study was to evaluate the feasibility, acceptability, and engagement of a single-session Zoom-delivered intervention for chronic pain. We also aimed to determine the preliminary efficacy of Zoom-delivered ER for reducing average pain intensity, pain catastrophizing, pain bothersomeness, and sleep disruption at 1-month posttreatment. Secondary and tertiary pain-related outcomes included physical function, anxiety, depression, and physical function. We also report a more rigorous test of the durability of treatment effects at 3-month posttreatment, as well as report the clinical meaningfulness of effects.

ER demonstrated high feasibility as indexed by high enrollment rate and excellent engagement; 94\% (47/50) of participants in the ER group attended the class and completed all surveys through the 3-month duration of the study. The enrollment rate was $53.1 \%$ (104/196), which is five times higher than that observed in other studies $[12,77]$ and stands as an index of both the inclusivity of the study protocol (few exclusionary criteria; all types of chronic pain) and the convenience of a single-session pain treatment study. Importantly, participants reported high overall satisfaction with the class (mean 8.26-8.77), the usefulness of presented information, and the likelihood of using the skills and information learned. In addition, participants reported high satisfaction with operating the Zoom platform (46/50, 92\% satisfaction), in addition to high satisfaction with engaging with class material, other attendees, and the class instructor. Importantly, of the 9 participants who indicated they had never used the Zoom platform during screening, none requested additional or individual training with the platform. These metrics indicate superior feasibility, accessibility, and engagement with the Zoom-delivered ER intervention.

With respect to preliminary efficacy, time effects showed reductions in pain intensity and most pain-related indices, except for sleep disruption. ER demonstrated superior treatment effects on average pain intensity and pain-related catastrophizing, pain bothersomeness, and sleep disruption over the 3 months following treatment. The between-groups Cohen $d$ effect sizes at 3-month posttreatment ranged from 0.60 to 0.91 for primary and secondary variables. For ER, pre- or posttreatment Cohen $d$ effect sizes ranged from 0.37 to 0.89 , demonstrating no clinical importance to moderate clinical importance for reducing pain-related catastrophizing, bothersomeness, and sleep disruption at the end of treatment. The treatment effect sizes across the primary and secondary outcomes suggested that a single-session, digital, skills-based intervention may have clinically meaningful effects on patient-reported outcomes. The durability of the treatment effects was demonstrated at the 3-month follow-up time-point. A greater proportion of participants in the ER group exceeded the thresholds for the clinical importance of effects at 3 months posttreatment.

First, for moderate importance in PCS reduction $(\geq 30 \%$ reduction in PCS), $62 \%$ (31/50) in ER versus 24\% (12/51) in 
WLC met this threshold. For substantial clinical importance in PCS reduction $(\geq 50 \%$ reduction in pain), $46 \%$ (23/50) in ER versus $12 \%(6 / 51)$ in WLC met this threshold. PCS reductions for ER (mean -8.72, SD 1.03) exceeded the clinically meaningful threshold of 6.8 reported in the literature [78] and is notably greater than that reported in other studies examining in-person 8-week CBT [7,10,79]. Our findings on PCS are expected and aligned with the literature suggesting that pain catastrophizing, an index of pain coping, is highly responsive to behavioral treatments $[7,10,79]$, including SSI $[11,12]$. For context, multidisciplinary pain rehabilitation research has shown that PCS reductions of $38 \%$ are clinically important and associated with less disability and work status at one-year follow-up [80].

Second, for minimal clinical importance in pain reduction $(\geq 15 \%$ reduction in pain), 30\% (15/50) in ER versus 6\% (3/51) in WLC met this threshold. The moderate clinical importance of pain reduction was low in both groups. Importantly, this study used a PROMIS pain measure with a stricter range (5-point scale) versus a traditional 11-point Numeric Pain Rating Scale. As such, the lack of variability in this measure may have negatively impacted our ability to detect the effects of pain intensity.

The tertiary outcomes yielded mixed findings. Reductions in anxiety and depression over time were found in both groups, and the decline was significantly greater for ER than WLC. However, these reductions did not reach the threshold for clinical significance. Notably, for depression, we observed regression to the mean at 3 months with the treatment effect lost, thus suggesting that more intensive treatment might be needed for durable reductions in depressive symptoms. This may be contextualized within widespread social isolation due to COVID-19, which has been shown to worsen depression symptoms over time $[81,82]$, or other stressors related to COVID-19 or the seasonal context of this study.

Interestingly, no treatment or time effects were observed for physical function, only a trend toward significance $(P=.09)$, which suggests that the study may be underpowered to detect differences or identify subgroups where effect may exist. In addition, due to a coding error, the short form used in this study did not capture upper extremity function, and scale items focused on lower mobility. As this was a mixed-etiology chronic pain study, comprehensive assessment of physical function is warranted to definitively explore its effects. Exploratory analyses revealed interaction effects for reductions in social isolation, which is a notable and important therapeutic target during the COVID-19 pandemic social restrictions. Although the two groups did not differ at 3 months, the interaction effect revealed a decrease in social isolation in the ER group but not in the WLC group. These findings lend support to ER serving as a buffer against the social threat perpetuated by the pandemic $[16,83]$, which can ameliorate its impact on people's overall health status $[65,66]$.

Although a growing body of research exists on the efficacy of ER for chronic and acute pain management [12,33,36,52], the use of Zoom as a platform to deliver behavioral medicine for chronic pain, particularly single-session treatment, remains novel and understudied. In addition, the extant literature on digital behavioral health research has reported participant treatment engagement rates ranging from $20 \%$ to $60 \%$ [33,84-86]. Strikingly, the current trial demonstrated a $94 \%$ (47/50) engagement rate in the treatment arm. These results also highlight the public interest in the web-based delivery of behavioral medicine interventions as a home-based chronic pain treatment modality. Combined with high participant engagement data and high satisfaction with using the Zoom platform, these data extend prior work [18-25] supporting the utility, user satisfaction, and efficacy of videoconference-delivered interventions for chronic pain.

\section{Strengths and Limitations}

Several findings should be considered within a number of limitations. First, given that the participant population was on average of higher socioeconomic status, as evidenced by their average education and income levels, the results may not be generalizable to populations of different demographics. No data were available on medical diagnoses, current prescriptions, over-the-counter medication use, or concurrent treatments for either study group. In addition, all data were self-reported, and we did not control for receipt of medical care. In light of COVID-19 restrictions, participants were likely to receive their care via web-based delivery as well. In addition, the pain intensity measure used was a 5-point Numeric Pain Rating Scale, as opposed to the 11-point Numeric Pain Rating Scale [87] typically used in research, limiting variability and may have obscured treatment effects and clinically meaningful findings. Finally, the participants were unblinded to the study group assignment. However, given that ER is low risk and low burden, placebo effects are less concerning. Replication studies were indicated with participants with more demographic variability over a longer follow-up period. Finally, future studies should characterize pain diagnoses and pain types.

Despite these limitations, this web-based study evidenced strong interest and participant engagement with single-session skills-based pain treatment, thus supporting further research and extension of web-based ER into clinical care. Second, the COVID-19 context and web-based delivery modality support the ecological validity of the study findings. Notably, the study was conducted remotely and did not benefit from any in-person contact that occurred when research was conducted in medical treatment settings (ie, halo effects). Additional aspects of methodological rigor included analyst blinding, intention-to-treat analyses, and randomization. Finally, our sample included minimal exclusions, which rendered it highly generalizable to other real-world individuals.

This study examined class cohorts comprising approximately 25 participants, and in-person class sizes ranged between 20 and 85, thus illustrating the promise of scalable pain care. Indeed, ER may help address pain disparities by ensuring rapid and more equitable access to pain treatment. Our findings provide initial evidence that the web-based delivery of ER may efficiently reduce the burden of chronic pain and improve symptom management. Additional research is needed to test the pragmatic comparative effectiveness of web-based ER to other treatments, such as gold-standard 8-week CBT, and to determine the heterogeneity of treatment effects. 


\section{Conclusions}

In 2019, the US Health and Human Services cited ER as a promising scalable behavioral pain treatment [88]. For the first time, this study determined the preliminary efficacy of the Zoom-delivered class in reducing the burden of chronic pain and improving symptom management. Importantly, the web-based single-session class addresses the rapidly expanding need for alternatives to face-to-face encounters due to the COVID-19 pandemic. Web-based delivery of ER stands to largely improve patient access and engagement because it is adaptable to medical or community settings, is readily extendable to underserved populations (eg, rural locations), and may be offered at a low cost.

\section{Acknowledgments}

The authors thank the National Institute on Drug Abuse for supporting this work: K23DA047473 (MSZ), K23DA048972 (DSY), and NCCIH R01AT008561 (BDD).

\section{Conflicts of Interest}

Stanford University receives revenue for empowered relief, continuing medical education and instructor certification training provided to health care clinicians. BDD is Chief Science Advisor at AppliedVR and her consulting role with this company (personal fees) is unrelated to the current research. BDD receives royalties for four pain treatment books she has authored or coauthored. BDD is also the principal investigator for a pain research award from the Patient-Centered Research Outcomes Research Institute and pain research grants from the National Institutes of Health. BDD received consultant fees from Axial Healthcare related to physician education for opioid prescribing and deprescribing (2019; unrelated to the current work). BDD serves on the Board of Directors for the American Academy of Pain Medicine and is on the Board of Directors for the Institute for Brain Potential. BDD is a scientific member of the National Institutes of Health Interagency Pain Research Coordinating Committee, the Centers for Disease Control and Prevention Opioid Workgroup and is a member of the Pain Advisory Group of the American Psychological Association.

\section{Multimedia Appendix 1}

Consent form.

[DOC File, 62 KB-Multimedia Appendix 1]

\section{Multimedia Appendix 2}

CONSORT-eHEALTH checklist (V 1.6.1).

[PDF File (Adobe PDF File), 277 KB-Multimedia Appendix 2]

\section{References}

1. Schappert SM, Burt CW. Ambulatory care visits to physician offices, hospital outpatient departments, and emergency departments: United States, 2001-02. Vital Health Stat 132006 Feb(159):1-66 [FREE Full text] [Medline: 16471269]

2. Institute of Medicine. Relieving Pain in America: A Blueprint for Transforming Prevention, Care, Education and Research. Washington, DC: The National Academies Press; 2011:55-112.

3. Becker N, Thomsen A, Olsen A, Sjøgren P, Bech P, Eriksen J. Pain epidemiology and health related quality of life in chronic non-malignant pain patients referred to a Danish multidisciplinary pain center. Pain 1997;73(3):393-400. [doi: 10.1016/S0304-3959(97)00126-7]

4. Shaheed CA, Maher CG, Williams KA, Day R, McLachlan AJ. Efficacy, tolerability, and dose-dependent effects of opioid analgesics for low back pain: a systematic review and meta-analysis. JAMA Intern Med 2016 Jul 01;176(7):958-968. [doi: 10.1001/jamainternmed.2016.1251] [Medline: 27213267]

5. Bernardy K, Füber N, Köllner V, Häuser W. Efficacy of cognitive-behavioral therapies in fibromyalgia syndrome - a systematic review and metaanalysis of randomized controlled trials. J Rheumatol 2010 Oct 03;37(10):1991-2005. [doi: 10.3899/jrheum.100104] [Medline: 20682676]

6. Darnall BD, Scheman J, Davin S, Burns JW, Murphy JL, Wilson AC, et al. Pain Psychology: A global needs assessment and national call to action. Pain Med 2016 Feb 23;17(2):250-263 [FREE Full text] [doi: 10.1093/pm/pnv095] [Medline: 26803844]

7. Williams ACC, Fisher E, Hearn L, Eccleston C. Psychological therapies for the management of chronic pain (excluding headache) in adults. Cochrane Database Syst Rev 2020 Aug 12;8(8):CD007407. [doi: 10.1002/14651858.CD007407.pub4] [Medline: $\underline{32794606]}$

8. Mariano TY, Urman RD, Hutchison CA, Jamison RN, Edwards RR. Cognitive behavioral therapy (CBT) for subacute low back pain: a systematic review. Curr Pain Headache Rep 2018 Feb 23;22(3):15. [doi: 10.1007/s11916-018-0669-5] [Medline: 29476270] 
9. Morley S, Eccleston C, Williams A. Systematic review and meta-analysis of randomized controlled trials of cognitive behaviour therapy and behaviour therapy for chronic pain in adults, excluding headache. Pain 1999;80(1):1-13. [doi: 10.1016/S0304-3959(98)00255-3]

10. Cherkin DC, Sherman KJ, Balderson BH, Cook AJ, Anderson ML, Hawkes RJ, et al. Effect of mindfulness-based stress reduction vs cognitive behavioral therapy or usual care on back pain and functional limitations in adults with chronic low back pain: a randomized clinical trial. J Am Med Assoc 2016 Mar 22;315(12):1240-1249 [FREE Full text] [doi: 10.1001/jama.2016.2323] [Medline: 27002445]

11. Darnall BD, Ziadni MS, Roy A, Kao MC, Sturgeon JA, Cook KF, et al. Comparative efficacy and mechanisms of a single-session pain psychology class in chronic low back pain: study protocol for a randomized controlled trial. Trials 2018 Mar 06;19(1):165 [FREE Full text] [doi: 10.1186/s13063-018-2537-3] [Medline: 29510735]

12. Darnall BD, Roy A, Chen AL, Ziadni MS, Keane RT, You DS, et al. Comparison of a single-session pain management skills intervention with a single-session health education intervention and 8 sessions of cognitive behavioral therapy in adults with chronic low back pain: a randomized clinical trial. JAMA Network Open 2021 Aug 16;4(8):e2113401 [FREE Full text] [doi: 10.1001/jamanetworkopen.2021.13401]

13. Earnshaw VA, Quinn DM. The impact of stigma in healthcare on people living with chronic illnesses. J Health Psychol 2012 Mar 28;17(2):157-168. [doi: 10.1177/1359105311414952] [Medline: 21799078]

14. Sharf J, Primavera LH, Diener MJ. Dropout and therapeutic alliance: a meta-analysis of adult individual psychotherapy. Psychotherapy (Chic) 2010 Dec;47(4):637-645. [doi: 10.1037/a0021175] [Medline: 21198249]

15. Eccleston C, Blyth FM, Dear BF, Fisher EA, Keefe FJ, Lynch ME, et al. Managing patients with chronic pain during the COVID-19 outbreak: considerations for the rapid introduction of remotely supported (eHealth) pain management services. Pain 2020 May;161(5):889-893 [FREE Full text] [doi: 10.1097/j.pain.0000000000001885] [Medline: $\underline{32251203]}$

16. Kazdin AE. Annual Research Review: Expanding mental health services through novel models of intervention delivery. J Child Psychol Psychiatry 2019 Apr 13;60(4):455-472. [doi: 10.1111/jcpp.12937] [Medline: 29900543]

17. Barak A, Hen L, Boniel-Nissim M, Shapira N. A comprehensive review and a meta-analysis of the effectiveness of internet-based psychotherapeutic interventions. J Technol Hum Serv 2008 Jul 03;26(2-4):109-160. [doi: 10.1080/15228830802094429]

18. Hedman E, Ljótsson B, Lindefors N. Cognitive behavior therapy via the internet: a systematic review of applications, clinical efficacy and cost-effectiveness. Expert Rev Pharmacoecon Outcomes Res 2012 Dec;12(6):745-764. [doi: 10.1586/erp.12.67] [Medline: 23252357]

19. Beatty L, Lambert S. A systematic review of internet-based self-help therapeutic interventions to improve distress and disease-control among adults with chronic health conditions. Clin Psychol Rev 2013 Jun;33(4):609-622. [doi: 10.1016/j.cpr.2013.03.004] [Medline: 23603521]

20. Cuijpers P, van Straten A, Andersson G. Internet-administered cognitive behavior therapy for health problems: a systematic review. J Behav Med 2008 Apr;31(2):169-177 [FREE Full text] [doi: 10.1007/s10865-007-9144-1] [Medline: 18165893]

21. Garg S, Garg D, Turin TC, Chowdhury MF. Web-based interventions for chronic back pain: a systematic review. J Med Internet Res 2016 Jul 26;18(7):e139 [FRE Full text] [doi: 10.2196/jmir.4932] [Medline: 27460413]

22. Dhokia M, Elander J, Clements K, Gilbert P. A randomized-controlled pilot trial of an online compassionate mind training intervention to help people with chronic pain avoid analgesic misuse. Psychol Addict Behav 2020 Nov;34(7):726-733. [doi: 10.1037/adb0000579] [Medline: 32271055]

23. Young SD, Koussa M, Lee S, Perez H, Gill N, Gelberg L, et al. Feasibility of a social media/online community support group intervention among chronic pain patients on opioid therapy. J Addict Dis 2018;37(1-2):96-101 [FREE Full text] [doi: 10.1080/10550887.2018.1557992] [Medline: 30614403]

24. Wilson M, Finlay M, Orr M, Barbosa-Leiker C, Sherazi N, Roberts ML, et al. Engagement in online pain self-management improves pain in adults on medication-assisted behavioral treatment for opioid use disorders. Addict Behav 2018 Nov;86:130-137. [doi: 10.1016/j.addbeh.2018.04.019] [Medline: 29731244]

25. Flynn N. Effect of an online hypnosis intervention in reducing migraine symptoms: a randomized controlled trial. Int J Clin Exp Hypn 2019 Jun 28;67(3):313-335. [doi: 10.1080/00207144.2019.1612674] [Medline: 31251706]

26. Kazdin AE, Blase SL. Interventions and models of their delivery to reduce the burden of mental illness: reply to commentaries. Perspect Psychol Sci 2011 Sep;6(5):507-510. [doi: 10.1177/1745691611418241] [Medline: 26168204]

27. Schleider JL, Weisz JR. Little treatments, promising effects? Meta-analysis of single-session interventions for youth psychiatric problems. J Am Acad Child Adolesc Psychiatry 2017 Feb;56(2):107-115. [doi: 10.1016/j.jaac.2016.11.007] [Medline: 28117056]

28. Depp C, Perivoliotis D, Holden J, Dorr J, Granholm E. Single-session mobile-augmented intervention in serious mental illness: a three-arm randomized controlled trial. Schizophr Bull 2019 Jun 18;45(4):752-762 [FREE Full text] [doi: 10.1093/schbul/sby135] [Medline: 30281086]

29. Ellis J, Cushing T, Germain A. Treating Acute Insomnia: A randomized controlled trial of a "Single-Shot" of cognitive behavioral therapy for insomnia. Sleep 2015 Jun 01;38(6):971-978 [FREE Full text] [doi: 10.5665/sleep.4752] [Medline: 25515106] 
30. Samson JE, Tanner-Smith EE. Single-session alcohol interventions for heavy drinking college students: a systematic review and meta-analysis. J Stud Alcohol Drugs 2015 Jul;76(4):530-543 [FREE Full text] [doi: 10.15288/jsad.2015.76.530] [Medline: 26098028]

31. Ziadni MS, Carty JN, Doherty HK, Porcerelli JH, Rapport LJ, Schubiner H, et al. A life-stress, emotional awareness, and expression interview for primary care patients with medically unexplained symptoms: a randomized controlled trial. Health Psychol 2018 Mar;37(3):282-290 [FREE Full text] [doi: 10.1037/hea0000566] [Medline: 29154608]

32. Carty JN, Ziadni MS, Holmes HJ, Tomakowsky J, Peters K, Schubiner H, et al. The effects of a life stress emotional awareness and expression interview for women with chronic urogenital pain: a randomized controlled trial. Pain Med 2019 Jul 01;20(7):1321-1329 [FREE Full text] [doi: 10.1093/pm/pny182] [Medline: $\underline{\text { 30252113] }}$

33. Darnall BD, Ziadni MS, Krishnamurthy P, Flood P, Heathcote LC, Mackey IG, et al. "My Surgical Success": Effect of a digital behavioral pain medicine intervention on time to opioid cessation after breast cancer surgery-a pilot randomized controlled clinical trial. Pain Med 2019 Nov 01;20(11):2228-2237 [FREE Full text] [doi: 10.1093/pm/pnz094] [Medline: 31087093]

34. Ziadni MS, Chen AL, Winslow T, Mackey SC, Darnall BD. Efficacy and mechanisms of a single-session behavioral medicine class among patients with chronic pain taking prescription opioids: study protocol for a randomized controlled trial. Trials 2020 Jun 12;21(1):521 [FREE Full text] [doi: 10.1186/s13063-020-04415-x] [Medline: 32532346]

35. Ziadni MS, Gonzalez-Castro LA, Darnall BD. Virtual single-session "Empowered Relief” group intervention for chronic pain. ClinicalTrials.gov. 2020 Sep 14. URL: https://clinicaltrials.gov/ct2/show/

NCT04546685?id=NCT04546685\&draw=2\&rank=1 [accessed 2021-08-09]

36. Darnall BD, Sturgeon JA, Kao MC, Hah JM, Mackey SC. From Catastrophizing to Recovery: a pilot study of a single-session treatment for pain catastrophizing. J Pain Res 2014;7:219-226 [FREE Full text] [doi: 10.2147/JPR.S62329] [Medline: 24851056]

37. Schleider JL, Dobias ML, Sung JY, Mullarkey MC. Future directions in single-session youth mental health interventions. J Clin Child Adolesc Psychol 2020 Dec 04;49(2):264-278 [FREE Full text] [doi: 10.1080/15374416.2019.1683852] [Medline: 31799863]

38. Alschuler KN, Altman JK, Ehde DM. Feasibility and acceptability of a single-session, videoconference-delivered group intervention for pain in multiple sclerosis. Rehabil Psychol 2021 Feb;66(1):22-30. [doi: 10.1037/rep0000360] [Medline: 32790454]

39. Rodda SN, Lubman DI, Jackson AC, Dowling NA. Improved outcomes following a single session web-based intervention for problem gambling. J Gambl Stud 2017 Mar;33(1):283-299. [doi: 10.1007/s10899-016-9638-2] [Medline: 27562272]

40. Schleider JL, Dobias M, Sung J, Mumper E, Mullarkey MC. Acceptability and utility of an open-access, online single-session intervention platform for adolescent mental health. JMIR Ment Health 2020 Jun 30;7(6):e20513 [FREE Full text] [doi: 10.2196/20513] [Medline: $\underline{32602846}$ ]

41. Wasil AR, Park SJ, Gillespie S, Shingleton R, Shinde S, Natu S, et al. Harnessing single-session interventions to improve adolescent mental health and well-being in India: Development, adaptation, and pilot testing of online single-session interventions in Indian secondary schools. Asian J Psychiatr 2020 Apr;50:101980. [doi: 10.1016/j.ajp.2020.101980] [Medline: 32146337]

42. Cardamone-Breen MC, Jorm AF, Lawrence KA, Rapee RM, Mackinnon AJ, Yap MB. A single-session, web-based parenting intervention to prevent adolescent depression and anxiety disorders: randomized controlled trial. J Med Internet Res 2018 Dec 26;20(4):e148 [FREE Full text] [doi: 10.2196/jmir.9499] [Medline: 29699964]

43. Hymmen P, Stalker CA, Cait C. The case for single-session therapy: Does the empirical evidence support the increased prevalence of this service delivery model? J Ment Health 2013 Feb 23;22(1):60-71. [doi: 10.3109/09638237.2012.670880] [Medline: 23343048]

44. Harris PA, Taylor R, Thielke R, Payne J, Gonzalez N, Conde JG. Research electronic data capture (REDCap)--a metadata-driven methodology and workflow process for providing translational research informatics support. J Biomed Inform 2009 Apr;42(2):377-381 [FREE Full text] [doi: 10.1016/j.jbi.2008.08.010] [Medline: 18929686]

45. Zoom video communications. Zoom. URL: https://stanford.zoom.us [accessed 2021-08-09]

46. Kaldo V, Levin S, Widarsson J, Buhrman M, Larsen H, Andersson G. Internet versus group cognitive-behavioral treatment of distress associated with tinnitus: a randomized controlled trial. Behav Ther 2008 Dec;39(4):348-359. [doi: 10.1016/j.beth.2007.10.003] [Medline: 19027431]

47. Riva S, Camerini A, Allam A, Schulz PJ. Interactive sections of an internet-based intervention increase empowerment of chronic back pain patients: randomized controlled trial. J Med Internet Res 2014 Aug;16(8):e180 [FREE Full text] [doi: 10.2196/jmir.3474] [Medline: 25119374]

48. Buhrman M, Fältenhag S, Ström L, Andersson G. Controlled trial of internet-based treatment with telephone support for chronic back pain. Pain 2004 Oct;111(3):368-377. [doi: 10.1016/j.pain.2004.07.021] [Medline: 15363881]

49. Brattberg G. Internet-based rehabilitation for individuals with chronic pain and burnout: a randomized trial. Int J Rehab Res 2006;29(3):221-227. [doi: 10.1097/01.mrr.0000210055.17291.f5] [Medline: 16900043] 
50. Ferguson RJ, Sigmon ST, Pritchard AJ, LaBrie SL, Goetze RE, Fink CM, et al. A randomized trial of videoconference-delivered cognitive behavioral therapy for survivors of breast cancer with self-reported cognitive dysfunction. Cancer 2016 Jun 01;122(11):1782-1791 [ [FREE Full text] [doi: 10.1002/cncr.29891] [Medline: 27135464]

51. Owen J, Klapow J, Roth D, Shuster J, Bellis J, Meredith R, et al. Randomized pilot of a self-guided internet coping group for women with early-stage breast cancer. Ann Behav Med 2005 Aug;30(1):54-64. [doi: 10.1207/s15324796abm3001 7] [Medline: 16097906]

52. Ziadni MS, Anderson SR, Gonzalez-Castro L, Darnall BD. Comparative efficacy of a single-session "Empowered Relief" videoconference-delivered group intervention for chronic pain: study protocol for a randomized controlled trial. Trials 2021 May 22;22(1):358 [FREE Full text] [doi: 10.1186/s13063-021-05303-8] [Medline: 34022930]

53. Empowered Relief (ER) Certification. Stanford University. URL: https://empoweredrelief.stanford.edu [accessed 2021-08-09]

54. Hart T, Fann JR, Novack TA. The dilemma of the control condition in experience-based cognitive and behavioural treatment research. Neuropsychol Rehabil 2008 Jan;18(1):1-21. [doi: 10.1080/09602010601082359] [Medline: 17852761]

55. Mohr DC, Spring B, Freedland KE, Beckner V, Arean P, Hollon SD, et al. The selection and design of control conditions for randomized controlled trials of psychological interventions. Psychother Psychosom 2009;78(5):275-284. [doi: 10.1159/000228248] [Medline: 19602916]

56. Sullivan MJ, Bishop SR, Pivik J. The Pain Catastrophizing Scale: Development and validation. Psychol Assess 1995;7(4):524-532. [doi: 10.1037/1040-3590.7.4.524]

57. Osman A, Barrios F, Gutierrez P, Kopper B, Merrifield T, Grittmann L. The pain catastrophizing scale: further psychometric evaluation with adult samples. J Behav Med 2000;23(4):351-365. [doi: 10.1023/A:1005548801037]

58. Turner JA, Anderson ML, Balderson BH, Cook AJ, Sherman KJ, Cherkin DC. Mindfulness-based stress reduction and cognitive behavioral therapy for chronic low back pain: similar effects on mindfulness, catastrophizing, self-efficacy, and acceptance in a randomized controlled trial. Pain 2016 Nov;157(11):2434-2444 [FREE Full text] [doi: 10.1097/j.pain.0000000000000635] [Medline: 27257859]

59. Hung M, Hon SD, Franklin JD, Kendall RW, Lawrence BD, Neese A, et al. Psychometric properties of the PROMIS physical function item bank in patients with spinal disorders. Spine (Phila Pa 1976) 2014 Jan 15;39(2):158-163. [doi: 10.1097/BRS.0000000000000097] [Medline: 24173018]

60. Karayannis N, Baumann I, Sturgeon J, Melloh M, Mackey S. The impact of social isolation on pain interference: a longitudinal study. Ann Behav Med 2019 Jan 01;53(1):65-74 [FREE Full text] [doi: 10.1093/abm/kay017] [Medline: 29668841]

61. Karayannis NV, Sturgeon JA, Chih-Kao M, Cooley C, Mackey SC. Pain interference and physical function demonstrate poor longitudinal association in people living with pain: a PROMIS investigation. Pain 2017 Jun;158(6):1063-1068 [FREE Full text] [doi: 10.1097/j.pain.0000000000000881] [Medline: 28221284]

62. Lai J, Cella D, Choi S, Junghaenel DU, Christodoulou C, Gershon R, et al. How item banks and their application can influence measurement practice in rehabilitation medicine: a PROMIS fatigue item bank example. Arch Phys Med Rehabil 2011 Oct;92(10 Suppl):20-27 [FREE Full text] [doi: 10.1016/j.apmr.2010.08.033] [Medline: 21958919]

63. Revicki D, Chen W, Harnam N, Cook K, Amtmann D, Callahan L, et al. Development and psychometric analysis of the PROMIS pain behavior item bank. Pain 2009 Nov;146(1-2):158-169 [FREE Full text] [doi: 10.1016/j.pain.2009.07.029] [Medline: 19683873$]$

64. Revicki DA, Cook KF, Amtmann D, Harnam N, Chen W, Keefe FJ. Exploratory and confirmatory factor analysis of the PROMIS pain quality item bank. Qual Life Res 2013 Jul 9;23(1):245-255. [doi: 10.1007/s11136-013-0467-9] [Medline: 23836435]

65. Sturgeon JA, Carriere JS, Kao MC, Rico T, Darnall BD, Mackey SC. Social disruption mediates the relationship between perceived injustice and anger in chronic pain: a collaborative health outcomes information registry study. Ann Behav Med 2016 Dec 20;50(6):802-812 [FREE Full text] [doi: 10.1007/s12160-016-9808-6] [Medline: 27325314]

66. Sturgeon JA, Dixon EA, Darnall BD, Mackey SC. Contributions of physical function and satisfaction with social roles to emotional distress in chronic pain: a Collaborative Health Outcomes Information Registry (CHOIR) study. Pain 2015 Dec;156(12):2627-2633 [FREE Full text] [doi: 10.1097/j.pain.0000000000000313] [Medline: 26230739]

67. Sturgeon JA, Darnall BD, Kao MC, Mackey SC. Physical and psychological correlates of fatigue and physical function: a Collaborative Health Outcomes Information Registry (CHOIR) study. J Pain 2015 Mar;16(3):291-298 [FREE Full text] [doi: 10.1016/j.jpain.2014.12.004] [Medline: 25536536]

68. Dworkin RH, Turk DC, Farrar JT, Haythornthwaite JA, Jensen MP, Katz NP, IMMPACT. Core outcome measures for chronic pain clinical trials: IMMPACT recommendations. Pain 2005 Jan;113(1-2):9-19. [doi: 10.1016/j.pain.2004.09.012] [Medline: 15621359 ]

69. Turk DC, Dworkin RH, Allen RR, Bellamy N, Brandenburg N, Carr DB, et al. Core outcome domains for chronic pain clinical trials: IMMPACT recommendations. Pain 2003 Dec;106(3):337-345. [doi: 10.1016/j.pain.2003.08.001] [Medline: 14659516]

70. PROMIS-Assessment. Assessment Center. URL: https://www.assessmentcenter.net/ [accessed 2021-08-09]

71. Gershon RC, Rothrock N, Hanrahan R, Bass M, Cella D. The use of PROMIS and assessment center to deliver patient-reported outcome measures in clinical research. J Appl Meas 2010;11(3):304-314 [FREE Full text] [Medline: 20847477] 
72. Fischer HF, Rose M. Scoring depression on a common metric: a comparison of EAP estimation, plausible value imputation, and full Bayesian IRT modeling. Multivariate Behav Res 2019 Sep 20;54(1):85-99. [doi: 10.1080/00273171.2018.1491381] [Medline: $\underline{\text { 30235003] }}$

73. Breivik H, Collett B, Ventafridda V, Cohen R, Gallacher D. Survey of chronic pain in Europe: prevalence, impact on daily life, and treatment. Eur J Pain 2006 May;10(4):287-333. [doi: 10.1016/j.ejpain.2005.06.009] [Medline: 16095934]

74. Demyttenaere K, Bruffaerts R, Lee S, Posada-Villa J, Kovess V, Angermeyer M, et al. Mental disorders among persons with chronic back or neck pain: results from the World Mental Health Surveys. Pain 2007 Jun;129(3):332-342. [doi: 10.1016/j.pain.2007.01.022] [Medline: 17350169]

75. Gore M, Sadosky A, Stacey BR, Tai K, Leslie D. The burden of chronic low back pain: clinical comorbidities, treatment patterns, and health care costs in usual care settings. Spine 2012;37(11):668-677. [doi: 10.1097/brs.0b013e318241e5de]

76. Tsang A, Von Korff M, Lee S, Alonso J, Karam E, Angermeyer MC, et al. Common chronic pain conditions in developed and developing countries: gender and age differences and comorbidity with depression-anxiety disorders. J Pain 2008 Oct;9(10):883-891. [doi: 10.1016/j.jpain.2008.05.005] [Medline: 18602869]

77. Garcia LM, Birckhead BJ, Krishnamurthy P, Sackman J, Mackey IG, Louis RG, et al. An 8-week self-administered at-home behavioral skills-based virtual reality program for chronic low back pain: double-blind, randomized, placebo-controlled trial conducted during COVID-19. J Med Internet Res 2021 Feb 22;23(2):e26292 [FREE Full text] [doi: 10.2196/26292] [Medline: 33484240]

78. Suzuki H, Aono S, Inoue S, Imajo Y, Nishida N, Funaba M, et al. Clinically significant changes in pain along the Pain Intensity Numerical Rating Scale in patients with chronic low back pain. PLoS One 2020 Mar 3;15(3):e0229228 [FREE Full text] [doi: 10.1371/journal.pone.0229228] [Medline: 32126108]

79. Cherkin DC, Sherman KJ, Balderson BH, Turner JA, Cook AJ, Stoelb B, et al. Comparison of complementary and alternative medicine with conventional mind-body therapies for chronic back pain: protocol for the Mind-body Approaches to Pain (MAP) randomized controlled trial. Trials 2014 Jun 07;15(1):211 [FREE Full text] [doi: 10.1186/1745-6215-15-211] [Medline: 24906419]

80. Scott W, Wideman T, Sullivan M. Clinically meaningful scores on pain catastrophizing before and after multidisciplinary rehabilitation: a prospective study of individuals with subacute pain after whiplash injury. Clin J Pain 2014 Mar;30(3):183-190. [doi: 10.1097/AJP.0b013e31828eee6c] [Medline: 23552561]

81. Ettman CK, Abdalla SM, Cohen GH, Sampson L, Vivier PM, Galea S. Prevalence of depression symptoms in US adults before and during the COVID-19 pandemic. JAMA Netw Open 2020 Sep 01;3(9):e2019686 [FREE Full text] [doi: 10.1001/jamanetworkopen.2020.19686] [Medline: $\underline{\text { 32876685] }}$

82. Twenge JM, Joiner TE. U.S. Census Bureau-assessed prevalence of anxiety and depressive symptoms in 2019 and during the 2020 COVID-19 pandemic. Depress Anxiety 2020 Oct;37(10):954-956 [FREE Full text] [doi: 10.1002/da.23077] [Medline: 32667081]

83. Karos K, McParland JL, Bunzli S, Devan H, Hirsh A, Kapos FP, et al. The social threats of COVID-19 for people with chronic pain. Pain 2020 Oct;161(10):2229-2235 [FREE Full text] [doi: 10.1097/j.pain.0000000000002004] [Medline: 32694381]

84. Geraghty AW, Torres LD, Leykin Y, Pérez-Stable EJ, Muñoz RF. Understanding attrition from international internet health interventions: a step towards global eHealth. Health Promot Int 2013 Sep 10;28(3):442-452 [FREE Full text] [doi: 10.1093/heapro/das029] [Medline: 22786673]

85. Geraghty AW, Wood AM, Hyland ME. Attrition from self-directed interventions: investigating the relationship between psychological predictors, intervention content and dropout from a body dissatisfaction intervention. Soc Sci Med 2010 Jul;71(1):30-37. [doi: 10.1016/j.socscimed.2010.03.007] [Medline: 20400220]

86. Eysenbach G. The law of attrition. J Med Internet Res 2005;7(1):e11 [FREE Full text] [doi: 10.2196/jmir.7.1.e11] [Medline: 15829473]

87. Farrar JT, Young JP, LaMoreaux L, Werth JL, Poole MR. Clinical importance of changes in chronic pain intensity measured on an 11-point numerical pain rating scale. Pain 2001;94(2):149-158. [doi: 10.1016/S0304-3959(01)00349-9]

88. U.S. Department of Health and Human Services. Pain Management Best Practices Inter-Agency Task Force Report: Updates, Gaps, Inconsistencies, and Recommendations. Washington, D.C: U. S. Department of Health and Human Services; 2019. URL: https://www.hhs.gov/sites/default/files/pmtf-final-report-2019-05-23.pdf [accessed 2021-08-09]
Abbreviations
CBT: cognitive behavioral therapy
CONSORT: Consolidated Standards of Reporting Trials
ER: empowered relief
HIPAA: Health Insurance Portability and Accountability Act
PCS: pain catastrophizing scale
PROMIS: Patient-Reported Outcomes Measurement Information System
REDCap: Research Electronic Data Capture 
SSI: single-session intervention

WLC: waitlist control

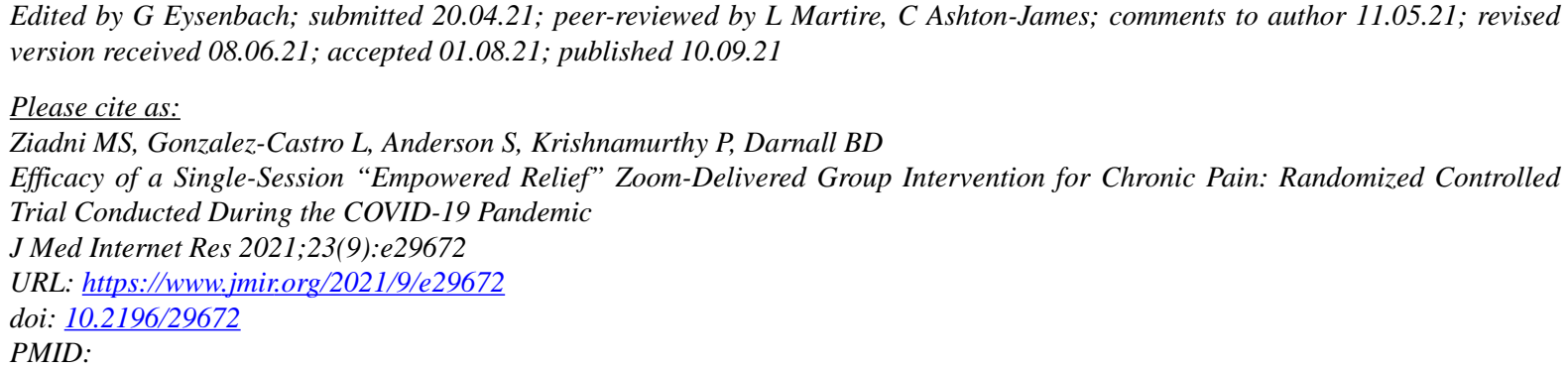

CMaisa S Ziadni, Lluvia Gonzalez-Castro, Steven Anderson, Parthasarathy Krishnamurthy, Beth D Darnall. Originally published in the Journal of Medical Internet Research (https://www.jmir.org), 10.09.2021. This is an open-access article distributed under the terms of the Creative Commons Attribution License (https://creativecommons.org/licenses/by/4.0/), which permits unrestricted use, distribution, and reproduction in any medium, provided the original work, first published in the Journal of Medical Internet Research, is properly cited. The complete bibliographic information, a link to the original publication on https://www.jmir.org/, as well as this copyright and license information must be included. 